\title{
Hadamard and Fejér-Hadamard Inequalities for Further Generalized Fractional Integrals Involving Mittag-Leffler Functions
}

\author{
M. Yussouf, ${ }^{1}$ G. Farid ${ }^{1 D},{ }^{2}$ K. A. Khan, ${ }^{1}$ and Chahn Yong Jung $\mathbb{D}^{3}$ \\ ${ }^{1}$ Department of Mathematics, University of Sargodha, Sargodha, Pakistan \\ ${ }^{2}$ Department of Mathematics, COMSATS University Islamabad, Attock Campus, Attock, Pakistan \\ ${ }^{3}$ Departmentof Business Administration, Gyeongsang National University, Jinju 52828, Republic of Korea \\ Correspondence should be addressed to G. Farid; faridphdsms@hotmail.com and Chahn Yong Jung; bb5734@gnu.ac.kr
}

Received 7 January 2021; Revised 19 February 2021; Accepted 24 February 2021; Published 16 March 2021

Academic Editor: Ahmet Ocak Akdemir

Copyright (c) 2021 M. Yussouf et al. This is an open access article distributed under the Creative Commons Attribution License, which permits unrestricted use, distribution, and reproduction in any medium, provided the original work is properly cited.

In this paper, generalized versions of Hadamard and Fejér-Hadamard type fractional integral inequalities are obtained. By using generalized fractional integrals containing Mittag-Leffler functions, some well-known results for convex and harmonically convex functions are generalized. The results of this paper are connected with various published fractional integral inequalities.

\section{Introduction}

First we give definitions of fractional integral operators which are useful in establishing the results of this paper. In the following, we give fractional integral operators defined by Andrić et al. in [1] via an extended generalized MittagLeffler function in their kernels.

Definition 1 (see [1]). Let $\omega, \tau, \delta, \rho, c \in \mathbb{C}, \mathfrak{R}(\tau), \mathfrak{R}(\delta)>0$, $\Re(c)>\mathfrak{R}(\rho)>0$ with $\bar{p} \geq 0, \sigma, r>0$ and $0<k \leq r+\sigma$. Let $\varphi \in L_{1}\left[\varepsilon_{1}, \varepsilon_{2}\right]$ and $x \in\left[\varepsilon_{1}, \varepsilon_{2}\right]$. Then, the generalized fractional integral operators $\epsilon_{\sigma, \tau, \delta, \omega, \varepsilon_{1}^{+}}^{\rho, r, k, c} \varphi$ and $\epsilon_{\sigma, \tau, \delta, \omega, \varepsilon_{2}}^{\rho, r, k, c} \varphi$ are defined by

$$
\begin{aligned}
& \left(\varepsilon_{\sigma, \tau, \delta, \omega, \varepsilon_{1}}^{\rho, r, k, c} \varphi\right)(x ; \bar{p})=\int_{\varepsilon_{1}}^{x}(x-t)^{\tau-1} E_{\sigma, \tau, \delta}^{\rho, r, k, c}\left(\omega(x-t)^{\sigma} ; \bar{p}\right) \varphi(t) \mathrm{d} t, \\
& \left(\varepsilon_{\sigma, \tau, \delta, \omega, \varepsilon_{2}}^{\rho, r, k, c} \varphi\right)(x ; \bar{p})=\int_{x}^{\varepsilon_{2}}(t-x)^{\tau-1} E_{\sigma, \tau, \delta}^{\rho, r, k, c}\left(\omega(t-x)^{\sigma} ; \bar{p}\right) \varphi(t) \mathrm{d} t,
\end{aligned}
$$

where

$$
E_{\sigma, \tau, \delta}^{\rho, r, k, c}(t ; \bar{p})=\sum_{n=0}^{\infty} \frac{\beta_{\bar{p}}(\rho+n k, c-\rho)(c)_{n k} t^{n}}{\beta(\rho, c-\rho) \Gamma(\sigma n+\tau)(\delta)_{n r}},
$$

is the extended generalized Mittag-Leffler function and $\beta_{\bar{p}}$ is the extension of beta function which is defined as follows:

$$
\beta_{\bar{p}}(x, y)=\int_{0}^{1} t^{x-1}(1-t)^{y-1} e^{-(\bar{p} / t(1-t))} \mathrm{d} t
$$

where $x, y, \bar{p}$ are positive real numbers.

Recently, Farid defined elegantly a unified integral operator in [2] (see, also [3]) as follows.

Definition 2. Let $\varphi, \theta:\left[\varepsilon_{1}, \varepsilon_{2}\right] \longrightarrow \mathbb{R}, 0<\varepsilon_{1}<\varepsilon_{2}$ be the functions such that $\varphi$ be positive and $\varphi \in L_{1}\left[\varepsilon_{1}, \varepsilon_{2}\right]$ and $\theta$ be a differentiable and strictly increasing function. Also, let $\chi / x$ be an increasing function on $\left[\varepsilon_{1}, \infty\right)$ and $\omega, \tau, \delta, \rho, c \in \mathbb{C}$, $\mathfrak{R}(\tau), \mathfrak{R}(\delta)>0, \mathfrak{R}(c)>\mathfrak{R}(\rho)>0$ with $\bar{p} \geq 0, \sigma, r>0$, and $0<k \leq r+\sigma$.

Then, for $x \in\left[\varepsilon_{1}, \varepsilon_{2}\right]$, the integral operators $\left({ }_{\theta} \Upsilon_{\sigma, \tau, \delta, \varepsilon_{1}^{+}}^{\chi, \rho, r, k} \varphi\right)$ and $\left({ }_{\theta} \Upsilon_{\sigma, \tau, \delta, \varepsilon_{1}^{-}}^{\chi}, \rho, r, k\right)$ are defined by 


$$
\begin{aligned}
& \left({ }_{\theta} \Upsilon_{\sigma, \tau, \delta, \varepsilon_{1}^{+}}^{\chi, \rho, r, k, c} \varphi\right)(x ; \bar{p})=\int_{\varepsilon_{1}}^{x} \frac{\chi(\theta(x)-\theta(t))}{\theta(x)-\theta(t)} E_{\sigma, \tau, \delta}^{\rho, r, k, c}\left(\omega(\theta(x)-\theta(t))^{\sigma} ; \bar{p}\right) \varphi(t) \mathrm{d}(\theta(t)), \\
& \left({ }_{\theta} \Upsilon_{\sigma, \tau, \delta, \varepsilon_{2}^{-}}^{\chi, \rho, r, k, c} \varphi\right)(x ; \bar{p})=\int_{x}^{\varepsilon_{2}} \frac{\chi(\theta(t)-\theta(x))}{\theta(t)-\theta(x)} E_{\sigma, \tau, \delta}^{\rho, r, k, c}\left(\omega(\theta(t)-\theta(x))^{\sigma} ; \bar{p}\right) \varphi(t) \mathrm{d}(\theta(t)) .
\end{aligned}
$$

The following definition of generalized fractional integral operators containing extended Mittag-Leffler function in the kernel can be extracted from Definition 2. It is generalization of Definition 1 by a monotonically increasing function.
Definition 3. Let $\varphi, \theta:\left[\varepsilon_{1}, \varepsilon_{2}\right] \longrightarrow \mathbb{R}, 0<\varepsilon_{1}<\varepsilon_{2}$ be the functions such that $\varphi$ be positive and $\varphi \in L_{1}\left[\varepsilon_{1}, \varepsilon_{2}\right]$ and $\theta$ be a differentiable and strictly increasing function. Also, let $\omega, \tau, \delta, \rho, c \in \mathbb{C}, \mathfrak{R}(\tau), \mathfrak{R}(\delta)>0, \mathfrak{R}(c)>\mathfrak{R}(\rho)>0$ with $\bar{p} \geq 0$, $\sigma, r>0$ and $0<k \leq r+\sigma$. Then, for $x \in\left[\varepsilon_{1}, \varepsilon_{2}\right]$, fractional integral operators are defined by

$$
\begin{aligned}
& \left({ }_{\theta} \Upsilon_{\sigma, \tau, \delta, \omega \varepsilon_{1}^{+}}^{\rho, r, k, c} \varphi\right)(x ; \bar{p})=\int_{\varepsilon_{1}}^{x}(\theta(x)-\theta(t))^{\tau-1} E_{\sigma, \tau, \delta}^{\rho, r, k, c}\left(\omega(\theta(x)-\theta(t))^{\sigma} ; \bar{p}\right) \varphi(t) \mathrm{d}(\theta(t)), \\
& \left({ }_{\theta} \Upsilon_{\sigma, \tau, \delta, \omega \varepsilon_{2}^{-}}^{\rho, r, k, c} \varphi\right)(x ; \bar{p})=\int_{x}^{\varepsilon_{2}}(\theta(t)-\theta(x))^{\tau-1} E_{\sigma, \tau, \delta}^{\rho, r, k, c}\left(\omega(\theta(t)-\theta(x))^{\sigma} ; \bar{p}\right) \varphi(t) \mathrm{d}(\theta(t)) .
\end{aligned}
$$

The following remark provides connection of Definition 3 with existing fractional integral operators.

\section{Remark 1}

(i) If we set $\bar{p}=0$ and $\theta(x)=x$ in equations (5) and (6), then these reduce to fractional integral operators defined by Salim and Faraj in [4].

(ii) If we set $\delta=r=1$ and $\theta(x)=x$ in equations (5) and (6), then these reduce to the fractional integral operators ${ }_{\theta} \Upsilon_{\sigma, \tau, 1, \omega, \varepsilon_{1}^{+}}^{\rho, 1, k,}$ and ${ }_{\theta} \Upsilon_{\sigma, \tau, 1, \omega, \varepsilon_{1}^{-}}^{\rho, 1, k, c}$ containing generalized Mittag-Leffler function $E_{\sigma, \tau, 1}^{\rho, 1, k, c}(t ; \bar{p})$ defined by Rahman et al. in [5].

(iii) If we take $\bar{p}=0, \delta=r=1$ and $\theta(x)=x$ in equations (5) and (6), then these reduce to fractional integral operators containing extended generalized Mittag-Leffler function introduced by Srivastava and Tomovski in [6].

(iv) If we set $\bar{p}=0, \delta=r=k=1$ and $\theta(x)=x$ in equations (5) and (6), then these reduce to fractional integral operators defined by Prabhaker in [7].

(v) For $\bar{p}=\omega=0$ and $\theta(x)=x$ in equations (5) and (6), these reduce to renowned Riemann-Liouville fractional integral operators [8].

The Riemann-Liouville fractional integrals for a function $\varphi \in L_{1}\left[\varepsilon_{1}, \varepsilon_{2}\right]$ of order $\tau \in \mathbb{R}(\tau>0)$ are defined by

$$
\begin{aligned}
& I_{\varepsilon_{1}^{+}}^{\tau} \varphi(x)=\frac{1}{\Gamma(\tau)} \int_{\varepsilon_{1}}^{x}(x-t)^{\tau-1} \varphi(t) \mathrm{d} t, \quad x>\varepsilon_{1}, \\
& I_{\varepsilon_{2}^{-}}^{\tau} \varphi(x)=\frac{1}{\Gamma(\tau)} \int_{x}^{\varepsilon_{2}}(t-x)^{\tau-1} \varphi(t) \mathrm{d} t, \quad x<\varepsilon_{2} .
\end{aligned}
$$

After introducing generalized fractional integral operators, now we define notions of functions for which generalized fractional integral operators are utilized to get main results of this paper.

Definition 4 (see [9]). A function $\varphi:\left[\varepsilon_{1}, \varepsilon_{2}\right] \longrightarrow \mathbb{R}$ is said to be convex if

$$
\varphi\left(t x_{1}+(1-t) x_{2}\right) \leq t \varphi\left(x_{1}\right)+(1-t) \varphi\left(x_{2}\right),
$$

holds for all $x_{1}, x_{2} \in\left[\varepsilon_{1}, \varepsilon_{2}\right]$ and $t \in[0,1]$.

Definition 5 (see [10]). Let $I$ be an interval such that $I \subseteq \mathbb{R}_{+}$. Then, a function $\varphi: I \longrightarrow \mathbb{R}$ is said to be harmonically convex, if

$$
\varphi\left(\frac{a b}{t a+(1-t) b}\right) \leq t \varphi(b)+(1-t) \varphi(a),
$$

holds for all $a, b \in I$ and $t \in[0,1]$.

Definition 6 (see [11]). Let $J \subset(0, \infty)$ be a real interval and $p \in \mathbb{R} \backslash\{0\}$. Then, a function $\varphi: J \longrightarrow \mathbb{R}$ is said to be $p$-convex, if

$$
\varphi\left(\left[t \varepsilon_{1}^{p}+(1-t) \varepsilon_{2}^{p}\right]^{1 / p}\right) \leq t \varphi\left(\varepsilon_{1}\right)+(1-t) \varphi\left(\varepsilon_{2}\right),
$$

holds for $\varepsilon_{1}, \varepsilon_{2} \in J$ and $t \in[0,1]$.

It is easy to see that for $p=1$ and $p=-1$, the $p$-convexity reduces to convexity and harmonical convexity, respectively.

Definition 7 (see $[11]$ ). Let $p \in \mathbb{R} \backslash\{0\}$. Then, a function $\varphi:\left[\varepsilon_{1}, \varepsilon_{2}\right] \subset(0, \infty) \longrightarrow \mathbb{R}$ is said to be $p$-symmetric with respect to $\left[\left(\varepsilon_{1}^{p}+\varepsilon_{2}^{p}\right) / 2\right]^{1 / p}$ if

$$
\varphi\left(t^{1 / p}\right)=\varphi\left(\left[\varepsilon_{1}^{p}+\varepsilon_{2}^{p}-t\right]^{1 / p}\right),
$$


holds, for $t \in\left[\varepsilon_{1}, \varepsilon_{2}\right]$.

Convex functions are equivalently studied by the Hadamard inequality.

Theorem 1. Let $\varphi:\left[\varepsilon_{1}, \varepsilon_{2}\right] \longrightarrow \mathbb{R}$ be a convex function such that $\varepsilon_{1}<\varepsilon_{2}$. Then, the following inequality holds:

$$
\varphi\left(\frac{\varepsilon_{1}+\varepsilon_{2}}{2}\right) \leq \frac{1}{\varepsilon_{2}-\varepsilon_{1}} \int_{\varepsilon_{1}}^{\varepsilon_{2}} \varphi(x) \mathrm{d} x \leq \frac{\varphi\left(\varepsilon_{1}\right)+\varphi\left(\varepsilon_{2}\right)}{2} .
$$

The Fejér-Hadamard inequality is a weighted version of the Hadamard inequality given by Fejér in [12].

Theorem 2. Let $\varphi:\left[\varepsilon_{1}, \varepsilon_{2}\right] \longrightarrow \mathbb{R}$ be a convex function and $g:\left[\varepsilon_{1}, \varepsilon_{2}\right] \longrightarrow \mathbb{R}$ be non-negative, integrable, and symmetric about $\left(\varepsilon_{1}+\varepsilon_{2}\right) / 2$. Then, the following inequality holds:

$$
\begin{aligned}
\varphi\left(\frac{\varepsilon_{1}+\varepsilon_{2}}{2}\right) \int_{\varepsilon_{1}}^{\varepsilon_{2}} g(x) \mathrm{d} x & \leq \int_{\varepsilon_{1}}^{\varepsilon_{2}} \varphi(x) g(x) \mathrm{d} x \\
& \leq \frac{\varphi\left(\varepsilon_{1}\right)+\varphi\left(\varepsilon_{2}\right)}{2} \int_{\varepsilon_{1}}^{\varepsilon_{2}} g(x) \mathrm{d} x .
\end{aligned}
$$

In recent decades, the Hadamard and the Fejér-Hadamard fractional integral inequalities have been studied extensively for different kinds of convex functions (see $[1,3,13-21])$. In this paper, we find Hadamard and Fejér-Hadamard inequalities for a generalized fractional integral operator involving an extended generalized MittagLeffler function.

In the upcoming section, we give two versions of the Hadamard inequality as well as two versions of the Fejér-Hadamard inequality. Their special cases are also discussed along with noticing connections with published results.

\section{Main Results}

First we give the following version of the Hadamard inequality.

Theorem 3. Let $\varphi, \theta:\left[\varepsilon_{1}, \varepsilon_{2}\right] \subset(0, \infty) \longrightarrow \mathbb{R}, \quad$ Range $(\theta) \subset\left[\varepsilon_{1}, \varepsilon_{2}\right]$ be the functions such that $\varphi$ be positive and $\varphi \in L_{1}\left[\varepsilon_{1}, \varepsilon_{2}\right]$, and $\theta$ be a differentiable and strictly increasing function. If $\varphi$ is $p$-convex, $p \in \mathbb{R} \backslash\{0\}$, then the following inequalities for fractional integral operators (5) and (6) hold:

(i) If $p>0$, then

$$
\begin{aligned}
& \varphi\left(\left(\frac{\theta^{p}\left(\varepsilon_{1}\right)+\theta^{p}\left(\varepsilon_{2}\right)}{2}\right)^{1 / p}\right)\left({ }_{\theta} \Upsilon_{\sigma, r, \delta, \omega^{\prime}, \theta^{-1}}^{\rho, r, k,}\left(\theta^{p}\left(\varepsilon_{1}\right)\right)_{+}{ }^{1}\right)\left(\theta^{-1}\left(\theta^{p}\left(\varepsilon_{2}\right)\right) ; \bar{p}\right) \\
& \leq \frac{1}{2}\left(\left({ }_{\theta} \Upsilon_{\sigma, \tau, \delta, \omega^{\prime}, \theta^{-1}\left(\theta^{p}\left(\varepsilon_{1}\right)\right)_{+}}^{\rho, r, k \circ \psi} \varphi\right)\left(\theta^{-1}\left(\theta^{p}\left(\varepsilon_{2}\right)\right) ; \bar{p}\right)\right. \\
& \left.+\left({ }_{\theta} Y_{\sigma, \tau, \delta, \omega^{\prime}, \theta^{-1}\left(\theta^{p}\left(\varepsilon_{2}\right)\right)_{-}}^{\rho, k, c} \varphi\right)\left(\theta^{-1}\left(\theta^{p}\left(\varepsilon_{1}\right)\right) ; \bar{p}\right)\right) \\
& \leq \frac{\varphi\left(\theta\left(\varepsilon_{1}\right)\right)+\varphi\left(\theta\left(\varepsilon_{2}\right)\right)}{2}\left({ }_{\theta} \Upsilon_{\sigma, \tau, \delta, \omega^{\prime}, \theta^{-1}\left(\theta^{p}\left(\varepsilon_{1}\right)\right)_{+}}^{\rho, k, c}\right)\left(\theta^{-1}\left(\theta^{p}\left(\varepsilon_{2}\right)\right) ; \bar{p}\right),
\end{aligned}
$$

where $\omega^{\prime}=\omega /\left(\theta^{p}\left(\varepsilon_{2}\right)-\theta^{p}\left(\varepsilon_{1}\right)\right)^{\sigma}$ and $\psi(t)=\theta^{1 / p}(t)$

for all $t \in\left[\varepsilon_{1}^{p}, \varepsilon_{2}^{p}\right]$.

(ii) If $p<0$, then

$$
\begin{aligned}
\varphi & \left(\left(\frac{\theta^{p}\left(\varepsilon_{1}\right)+\theta^{p}\left(\varepsilon_{2}\right)}{2}\right)^{1 / p}\right)\left(\varepsilon_{\sigma, \tau, \delta, \omega^{\prime}, \theta^{-1}}^{\rho, r, k, c}\left(\theta^{p}\left(\varepsilon_{1}\right)\right)_{-} 1\right)\left(\theta^{-1}\left(\theta^{p}\left(\varepsilon_{2}\right)\right) ; \bar{p}\right) \\
\leq & \frac{1}{2}\left(\left({ }_{\theta} \Upsilon_{\sigma, \tau, \delta, \omega^{\prime}, \theta^{-1}\left(\theta^{p}\left(\varepsilon_{2}\right)\right)_{+}}^{\rho, r, k, c} \psi\right)\left(\theta^{-1}\left(\theta^{p}\left(\varepsilon_{1}\right)\right) ; \bar{p}\right)\right. \\
& \left.+\left({ }_{\theta} \Upsilon_{\sigma, \tau, \delta, \omega^{\prime}, \theta^{-1}\left(\theta^{p}\left(\varepsilon_{1}\right)\right)_{-}}^{\rho, r, k, c} \varphi \psi\right)\left(\theta^{-1}\left(\theta^{p}\left(\varepsilon_{2}\right)\right) ; \bar{p}\right)\right) \\
\leq & \frac{\varphi\left(\theta\left(\varepsilon_{1}\right)\right)+\varphi\left(\theta\left(\varepsilon_{2}\right)\right)}{2}\left({ }_{\theta} \Upsilon_{\sigma, \tau, \delta, \omega^{\prime}, \theta^{-1}\left(\theta^{p}\left(\varepsilon_{1}\right)\right)_{-}}^{\rho, r, k, c}\right)\left(\theta^{-1}\left(\theta^{p}\left(\varepsilon_{2}\right)\right) ; \bar{p}\right),
\end{aligned}
$$

where $\omega^{\prime}=\omega /\left(\theta^{p}\left(\varepsilon_{1}\right)-\theta^{p}\left(\varepsilon_{2}\right)\right)^{\sigma}$ and $\psi(t)=\theta^{1 / p}(t)$ for all $t \in\left[\varepsilon_{2}^{p}, \varepsilon_{1}^{p}\right]$.
Proof. (i) Since $\varphi$ is $p$-convex over $\left[\varepsilon_{1}, \varepsilon_{2}\right]$, for all $x, y \in I$, we have 


$$
\varphi\left(\left(\frac{\theta^{p}(x)+\theta^{p}(y)}{2}\right)^{1 / p}\right) \leq \frac{\varphi(\theta(x))+\varphi(\theta(y))}{2}
$$

Setting $\theta(x)=\left(t \theta^{p}\left(\varepsilon_{1}\right)+(1-t) \theta^{p}\left(\varepsilon_{2}\right)\right)^{1 / p}$ and $\theta(y)=$ $\left(t \theta^{p}\left(\varepsilon_{2}\right)+(1-t) \theta^{p}\left(\varepsilon_{1}\right)\right)^{1 / p}$ in above inequality, we have

$$
\begin{aligned}
& \varphi\left(\left(\frac{\theta^{p}\left(\varepsilon_{1}\right)+\theta^{p}\left(\varepsilon_{2}\right)}{2}\right)^{1 / p}\right) \\
& \leq \frac{\varphi\left(\left(t \theta^{p}\left(\varepsilon_{1}\right)+(1-t) \theta^{p}\left(\varepsilon_{2}\right)\right)^{1 / p}\right)+\varphi\left(\left(t \theta^{p}\left(\varepsilon_{2}\right)+(1-t) \theta^{p}\left(\varepsilon_{1}\right)\right)^{1 / p}\right)}{2} .
\end{aligned}
$$

Multiplying both sides of (17) by $2 t^{\tau-1} E_{\sigma, \tau, \delta}^{\rho, r, k, c}\left(\omega t^{\sigma} ; \bar{p}\right)$ and then integrating over $[0,1]$, we have

$$
\begin{aligned}
& 2 \varphi\left(\left(\frac{\theta^{p}\left(\varepsilon_{1}\right)+\theta^{p}\left(\varepsilon_{2}\right)}{2}\right)^{1 / p}\right) \int_{0}^{1} t^{\tau-1} E_{\sigma, \tau, \delta}^{\rho, r, k, c}\left(\omega t^{\sigma} ; \bar{p}\right) \mathrm{d} t \\
& \leq \int_{0}^{1} t^{\tau-1} E_{\sigma, \tau, \delta}^{\rho, r, k, c}\left(\omega t^{\sigma} ; \bar{p}\right) \varphi\left(\left(t \theta^{p}\left(\varepsilon_{1}\right)+(1-t) \theta^{p}\left(\varepsilon_{2}\right)\right)^{1 / p}\right) \mathrm{d} t \\
& \quad+\int_{0}^{1} t^{\tau-1} E_{\sigma, \tau, \delta}^{\rho, r, k, c}\left(\omega t^{\sigma} ; \bar{p}\right) \varphi\left(\left(t \theta^{p}\left(\varepsilon_{2}\right)+(1-t) \theta^{p}\left(\varepsilon_{1}\right)\right)^{1 / p}\right) \mathrm{d} t .
\end{aligned}
$$

By choosing $\theta(x)=t \theta^{p}\left(\varepsilon_{1}\right)+(1-t) \theta^{p}\left(\varepsilon_{2}\right)$ and $\theta(y)=$ $t \theta^{p}\left(\varepsilon_{2}\right)+(1-t) \theta^{p}\left(\varepsilon_{1}\right)$ in (18), we have

$$
\begin{aligned}
& 2 \varphi\left(\left(\frac{\theta^{p}\left(\varepsilon_{1}\right)+\theta^{p}\left(\varepsilon_{2}\right)}{2}\right)^{1 / p}\right) \int_{\theta^{-1}\left(\theta^{p}\left(\varepsilon_{1}\right)\right)}^{\theta^{-1}\left(\theta^{p}\left(\varepsilon_{2}\right)\right)}\left(\theta^{p}\left(\varepsilon_{2}\right)-\theta(x)\right)^{\tau-1} E_{\sigma, \tau, \delta}^{\rho, r, k, c}\left(\omega^{\prime}\left(\theta^{p}\left(\varepsilon_{2}\right)-\theta(x)\right)^{\sigma} ; \bar{p}\right) 1 \mathrm{~d}(\theta(x)) \\
& \leq \int_{\theta^{-1}\left(\theta^{p}\left(\varepsilon_{1}\right)\right)}^{\theta^{-1}\left(\theta^{p}\left(\varepsilon_{2}\right)\right)}\left(\theta^{p}\left(\varepsilon_{2}\right)-\theta(x)\right)^{\tau-1} E_{\sigma, \tau, \delta}^{\rho, r, k, c}\left(\omega^{\prime}\left(\theta^{p}\left(\varepsilon_{2}\right)-\theta(x)\right)^{\sigma} ; \bar{p}\right) \varphi\left(\theta(x)^{1 / p}\right) \mathrm{d}(\theta(x)) \\
& \quad+\int_{\theta^{-1}\left(\theta^{p}\left(\varepsilon_{1}\right)\right)}^{\theta^{-1}\left(\theta^{p}\left(\varepsilon_{2}\right)\right)}\left(\theta(y)-\theta^{p}\left(\varepsilon_{1}\right)\right)^{\tau-1} E_{\sigma, \tau, \delta}^{\rho, r, k, c}\left(\omega^{\prime}\left(\theta(y)-\theta^{p}\left(\varepsilon_{1}\right)\right)^{\sigma} ; \bar{p}\right) \varphi\left(\theta(y)^{1 / p}\right) \mathrm{d}(\theta(y)),
\end{aligned}
$$

where $\omega^{\prime}=\omega /\left(\theta^{p}\left(\varepsilon_{2}\right)-\theta^{p}\left(\varepsilon_{1}\right)\right)^{\sigma}$.

This implies

$$
\begin{aligned}
& 2 \varphi\left(\left(\frac{\theta^{p}\left(\varepsilon_{1}\right)+\theta^{p}\left(\varepsilon_{2}\right)}{2}\right)^{1 / p}\right)\left(\varepsilon_{\sigma, \tau, \delta, \omega^{\prime}, \theta^{-1}\left(\theta^{p}\left(\varepsilon_{1}\right)\right)_{+}} 1\right)\left(\theta^{-1}\left(\theta^{p}\left(\varepsilon_{2}\right)\right) ; \bar{p}\right) \\
& \leq\left({ }_{\theta} \Upsilon_{\sigma, \tau, \delta, \omega^{\prime}, \theta^{-1}\left(\theta^{p}\left(\varepsilon_{1}\right)\right)_{+}}^{\rho, r, k, c} \varphi \circ \psi\right)\left(\theta^{-1}\left(\theta^{p}\left(\varepsilon_{2}\right)\right) ; \bar{p}\right) \\
& +\left({ }_{\theta} Y_{\sigma, \tau, \delta, \omega^{\prime}, \theta^{-1}\left(\theta^{p}\left(\varepsilon_{2}\right)\right)_{-}, r, k, c} \varphi \circ \psi\right)\left(\theta^{-1}\left(\theta^{p}\left(\varepsilon_{1}\right)\right) ; \bar{p}\right) .
\end{aligned}
$$

To prove the second inequality of (14), again from $p$-convexity of $\varphi$ over $\left[\varepsilon_{1}, \varepsilon_{2}\right]$ and for $t \in[0,1]$, we have

$$
\begin{aligned}
& \varphi\left(\left(t \theta^{p}\left(\varepsilon_{1}\right)+(1-t) \theta^{p}\left(\varepsilon_{2}\right)\right)^{1 / p}\right) \\
& \quad+\varphi\left(\left(t \theta^{p}\left(\varepsilon_{2}\right)+(1-t) \theta^{p}\left(\varepsilon_{1}\right)\right)^{1 / p}\right) \leq \varphi\left(\theta\left(\varepsilon_{1}\right)\right)+\varphi\left(\theta\left(\varepsilon_{2}\right)\right) .
\end{aligned}
$$

Multiplying both sides of $(21)$ by $t^{\tau-1} E_{\sigma, \tau, \delta}^{\rho, r, k, c}\left(\omega t^{\sigma} ; \bar{p}\right)$ and then integrating over $[0,1]$, we have

$$
\begin{aligned}
& \int_{0}^{1} t^{\tau-1} E_{\sigma, \tau, \delta}^{\rho, r, k, c}\left(\omega t^{\sigma} ; \bar{p}\right) \varphi\left(\left(t \theta^{p}\left(\varepsilon_{1}\right)+(1-t) \theta^{p}\left(\varepsilon_{2}\right)\right)^{1 / p}\right) \mathrm{d} t \\
& +\int_{0}^{1} t^{\tau-1} E_{\sigma, \tau, \delta}^{\rho, r, k, c}\left(\omega t^{\sigma} ; \bar{p}\right) \varphi\left(\left(t \theta^{p}\left(\varepsilon_{2}\right)+(1-t) \theta^{p}\left(\varepsilon_{1}\right)\right)^{1 / p}\right) \mathrm{d} t \\
& \leq\left(\varphi\left(\theta\left(\varepsilon_{1}\right)\right)+\varphi\left(\theta\left(\varepsilon_{2}\right)\right)\right) \int_{0}^{1} t^{\tau-1} E_{\sigma, \tau, \delta}^{\rho, r, k, c}\left(\omega t^{\sigma} ; \bar{p}\right) \mathrm{d} t
\end{aligned}
$$

Setting $\quad \theta(x)=t \theta^{p}\left(\varepsilon_{1}\right)+(1-t) \theta^{p}\left(\varepsilon_{2}\right)$ $\theta(y)=t \theta^{p}\left(\varepsilon_{2}\right)+(1-t) \theta^{p}\left(\varepsilon_{1}\right)$ in (22), we have 


$$
\begin{aligned}
& \left({ }_{\theta} \Upsilon_{\sigma, \tau, \delta, \omega^{\prime}, \theta^{-1}\left(\theta^{p}\left(\varepsilon_{1}\right)\right)_{+}, r, k \circ \psi} \varphi \circ\left(\theta^{-1}\left(\theta^{p}\left(\varepsilon_{2}\right)\right) ; \bar{p}\right)\right. \\
& \quad+\left({ }_{\theta} \Upsilon_{\sigma, \tau, \delta, \omega^{\prime}, \theta^{-1}\left(\theta^{p}\left(\varepsilon_{2}\right)\right)_{-}}^{\rho, r, c, c} \psi\right)\left(\theta^{-1}\left(\theta^{p}\left(\varepsilon_{1}\right)\right) ; \bar{p}\right) \\
& \leq\left(\varphi\left(\theta\left(\varepsilon_{1}\right)\right)+\varphi\left(\theta\left(\varepsilon_{2}\right)\right)\right) \\
& \quad \cdot\left({ }_{\theta} \Upsilon_{\sigma, \tau, \tau, \delta, \omega^{\prime}, \theta^{-1}\left(\theta^{p}\left(\varepsilon_{1}\right)\right)_{+}}^{\rho, r}\right)\left(\theta^{-1}\left(\theta^{p}\left(\varepsilon_{2}\right)\right) ; \bar{p}\right) .
\end{aligned}
$$

By combining (20) and (23), we get (14).

(ii) Proof is similar to the proof of (i).

Remark 2

(i) By setting $\bar{p}=\omega=0$ and $\theta=I$, Theorem 9 of [11] is obtained.

(ii) By setting $\bar{p}=0, p=-1$, and $\theta=I$, Theorem 2.1 of [22] is obtained.

(iii) By setting $\theta=I$ and $p=-1$, Theorem 2.1 of [23] is obtained.

(iv) By setting $\omega=\bar{p}=0, p=-1$, and $\theta=I$, Theorem 4 of [18] is obtained.

(v) By setting $p=-1$, Theorem 2.1 of [24] is obtained.

(vi) By setting $p=-1$ and $\psi(x)=x$, Corollary 2.3 of [24] is obtained.

Corollary 1. In (15), if we take $\omega=\bar{p}=0, p=-1$, and $\theta=I$, then we get the following Hadamard inequality for the $R L$ fractional integrals:

$$
\begin{aligned}
& \varphi\left(\frac{2 \varepsilon_{1} \varepsilon_{2}}{\varepsilon_{1}+\varepsilon_{2}}\right) \leq \frac{\Gamma(\tau+1)}{2}\left(\frac{\varepsilon_{1} \varepsilon_{2}}{\varepsilon_{2}-\varepsilon_{1}}\right)^{\tau} \\
& \cdot\left(\left(I_{1 \backslash \varepsilon_{1}-}^{\tau} \varphi \circ \psi\right)\left(\frac{1}{\varepsilon_{2}}\right)+\left(I_{1 \backslash \varepsilon_{1}+}^{\tau} \varphi \circ \psi\right)\left(\frac{1}{\varepsilon_{1}}\right)\right) \\
& \leq \frac{\varphi\left(\varepsilon_{1}\right)+\varphi\left(\varepsilon_{2}\right)}{2} .
\end{aligned}
$$

Now we obtain Fejér-Hadamard type fractional integral inequalities for $p$-convex function via generalized fractional integral operators; for this, first we prove the following lemma.

Lemma 1. Let $\varphi, \theta:\left[\varepsilon_{1}, \varepsilon_{2}\right] \subset(0, \infty) \longrightarrow \mathbb{R}, \quad$ Range $(\theta) \subset\left[\varepsilon_{1}, \varepsilon_{2}\right]$ be the functions such that $\varphi$ be positive and $\varphi \in L_{1}\left[\varepsilon_{1}, \varepsilon_{2}\right], \varepsilon_{1}<\varepsilon_{2}$, and $\theta$ be a differentiable and strictly increasing function. If $\varphi$ is $p$-convex, $p \in \mathbb{R} \backslash\{0\}$, and $\varphi\left(\theta^{1 / p}(x)\right)=\varphi\left(\left(\theta^{p}\left(\varepsilon_{1}\right)+\theta^{p}\left(\varepsilon_{2}\right)-\theta(x)\right)^{1 / p}\right)$, then for generalized fractional integral operators (5) and (6), we have

(i) If $p>0$, then

$$
\begin{aligned}
& \left({ }_{\theta} \Upsilon_{\sigma, \tau, \delta, \omega, \theta^{-1}\left(\theta^{p}\left(\varepsilon_{1}\right)\right)_{+}, r, k, c} \varphi \circ \psi\right)\left(\theta^{-1}\left(\theta^{p}\left(\varepsilon_{2}\right)\right) ; \bar{p}\right) \\
& =\left({ }_{\theta} \Upsilon_{\sigma, \tau, \delta, \omega, \theta^{-1}\left(\theta^{p}\left(\varepsilon_{2}\right)\right)_{-}}^{\rho, r, c} \varphi \circ \psi\right)\left(\theta^{-1}\left(\theta^{p}\left(\varepsilon_{1}\right)\right) ; \bar{p}\right) \\
& =\frac{1}{2}\left(\left({ }_{\theta} \Upsilon_{\sigma, \tau, \delta, \omega, \theta^{-1}\left(\theta^{p}\left(\varepsilon_{1}\right)\right)_{+}, r, k, c} \varphi \circ \psi\right)\left(\theta^{-1}\left(\theta^{p}\left(\varepsilon_{2}\right)\right) ; \bar{p}\right)\right. \\
& \left.+\left({ }_{\theta} \Upsilon_{\sigma, \tau, \delta, \omega, \theta^{-1}\left(\theta^{p}\left(\varepsilon_{2}\right)\right)_{-}}^{\rho, r, k, c} \varphi\right)\left(\theta^{-1}\left(\theta^{p}\left(\varepsilon_{1}\right)\right) ; \bar{p}\right)\right),
\end{aligned}
$$

with $\psi(t)=\theta^{1 / p}(t), t \in\left[\varepsilon_{1}^{p}, \varepsilon_{2}^{p}\right]$.

(ii) If $p<0$, then

$$
\begin{aligned}
& \left({ }_{\theta} \Upsilon_{\sigma, \tau, \delta, \omega, \theta^{-1}\left(\theta^{p}\left(\varepsilon_{2}\right)\right)_{+}}^{\rho, r, k, c} \varphi \circ \psi\right)\left(\theta^{-1}\left(\theta^{p}\left(\varepsilon_{1}\right)\right) ; \bar{p}\right) \\
& =\left({ }_{\theta} \Upsilon_{\sigma, \tau, \delta, \omega, \theta^{-1}\left(\theta^{p}\left(\varepsilon_{1}\right)\right)_{-}, r, k, c} \varphi \circ \psi\right)\left(\theta^{-1}\left(\theta^{p}\left(\varepsilon_{2}\right)\right) ; \bar{p}\right) \\
& =\frac{1}{2}\left(\left({ }_{\theta} \Upsilon_{\sigma, \tau, \delta, \omega, \theta^{-1}\left(\theta^{p}\left(\varepsilon_{2}\right)\right)_{+}}^{\rho, r, k, c} \varphi \circ \psi\right)\left(\theta^{-1}\left(\theta^{p}\left(\varepsilon_{1}\right)\right) ; \bar{p}\right)\right. \\
& \left.+\left({ }_{\theta} \Upsilon_{\sigma, \tau, \delta, \omega^{\prime}, \theta^{-1}\left(\theta^{p}\left(\varepsilon_{1}\right)\right)_{-}}^{\rho, r, k, c}{ }^{\prime} \circ \psi\right)\left(\theta^{-1}\left(\theta^{p}\left(\varepsilon_{2}\right)\right) ; \bar{p}\right)\right),
\end{aligned}
$$

with $\psi(t)=\theta^{1 / p}(t), t \in\left[\varepsilon_{2}^{p}, \varepsilon_{1}^{p}\right]$.

Proof. (i) By definition of generalized fractional integral operators (5) and (6), we have

$$
\begin{aligned}
& \left(\Upsilon_{\theta, \tau, \delta, \omega, \theta^{-1}\left(\theta^{p}\left(\varepsilon_{1}\right)\right)_{+}^{\rho, r, k}}^{\rho, c} \psi\right)\left(\theta^{-1}\left(\theta^{p}\left(\varepsilon_{2}\right)\right) ; \bar{p}\right) \\
& =\int_{\theta^{-1}\left(\theta^{p}\left(\varepsilon_{1}\right)\right)}^{\theta^{-1}\left(\theta^{p}\left(\varepsilon_{2}\right)\right)}\left(\theta^{p}\left(\varepsilon_{2}\right)-\theta(x)\right)^{\tau-1} E_{\sigma, \tau, \delta}^{\rho, r, k, c}\left(\omega\left(\theta^{p}\left(\varepsilon_{2}\right)-\theta(x)\right)^{\sigma} ; \bar{p}\right) \varphi \circ \psi(x) \mathrm{d}(\theta(x)) \\
& =\int_{\theta^{-1}\left(\theta^{p}\left(\varepsilon_{1}\right)\right)}^{\theta^{-1}\left(\theta^{p}\left(\varepsilon_{2}\right)\right)}\left(\theta^{p}\left(\varepsilon_{2}\right)-\theta(x)\right)^{\tau-1} E_{\sigma, \tau, \delta}^{\rho, r, k, c}\left(\omega\left(\theta^{p}\left(\varepsilon_{2}\right)-\theta(x)\right)^{\sigma} ; \bar{p}\right) \varphi\left(\theta(x)^{1 / p}\right) \mathrm{d}(\theta(x)) \\
& =\int_{\theta^{-1}\left(\theta^{p}\left(\varepsilon_{1}\right)\right)}^{\theta^{-1}\left(\theta^{p}\left(\varepsilon_{2}\right)\right)}\left(\theta^{p}\left(\varepsilon_{2}\right)-\theta(x)\right)^{\tau-1} E_{\sigma, \tau, \delta}^{\rho, r, k, c}\left(\omega\left(\theta^{p}\left(\varepsilon_{2}\right)-\theta(x)\right)^{\sigma} ; \bar{p}\right) \varphi\left(\left(\theta^{p}\left(\varepsilon_{1}\right)+\theta^{p}\left(\varepsilon_{2}\right)-\theta(x)\right)^{1 / p}\right) \mathrm{d}(\theta(x))
\end{aligned}
$$


Setting $\theta(t)=\theta^{p}\left(\varepsilon_{1}\right)+\theta^{p}\left(\varepsilon_{2}\right)-\theta(x)$ in the above equation and using $\varphi\left(\theta^{1 / p}(x)\right)=\varphi\left(\left(\theta^{p}\left(\varepsilon_{1}\right)+\right.\right.$ $\left.\left.\theta^{p}\left(\varepsilon_{2}\right)-\theta(x)\right)^{1 / p}\right)$, we have

$$
\begin{aligned}
& \left({ }_{\theta} \Upsilon_{\sigma, \tau, \delta, \omega, \theta^{-1}\left(\theta^{p}\left(\varepsilon_{1}\right)\right)_{+}}^{\rho, r, k, c} \varphi\right)\left(\theta^{-1}\left(\theta^{p}\left(\varepsilon_{2}\right)\right) ; \bar{p}\right) \\
& =\int_{\theta^{-1}\left(\theta^{p}\left(\varepsilon_{1}\right)\right)}^{\theta^{-1}\left(\theta^{p}\left(\varepsilon_{2}\right)\right)}\left(\theta(t)-\theta^{p}\left(\varepsilon_{1}\right)\right)^{\tau-1} E_{\sigma, \tau, \delta}^{\rho, r, k, c}\left(\omega\left(\theta(t)-\theta^{p}\left(\varepsilon_{1}\right)\right)^{\sigma} ; \bar{p}\right) \varphi\left(\theta(t)^{1 / p}\right) \mathrm{d}(\theta(t)) \\
& =\int_{\theta^{-1}\left(\theta^{p}\left(\varepsilon_{1}\right)\right)}^{\theta^{-1}\left(\theta^{p}\left(\varepsilon_{2}\right)\right)}\left(\theta(t)-\theta^{p}\left(\varepsilon_{1}\right)\right)^{\tau-1} E_{\sigma, \tau, \delta}^{\rho, r, k, c}\left(\omega\left(\theta(t)-\theta^{p}\left(\varepsilon_{1}\right)\right)^{\sigma} ; \bar{p}\right) \varphi \circ \psi(t) \mathrm{d}(\theta(t)) .
\end{aligned}
$$

This implies

$$
\begin{aligned}
& \left({ }_{\theta} \Upsilon_{\sigma, \tau, \delta, \omega, \theta^{-1}\left(\theta^{p}\left(\varepsilon_{1}\right)\right)_{+}}^{\rho, r, k, c} \varphi \circ \psi\right)\left(\theta^{-1}\left(\theta^{p}\left(\varepsilon_{2}\right)\right) ; \bar{p}\right) \\
& =\left({ }_{\theta} \Upsilon_{\sigma, \tau, \delta, \omega, \theta^{-1}\left(\theta^{p}\left(\varepsilon_{2}\right)\right)_{-}}^{\rho, r, k, c} \varphi \psi \psi\left(\theta^{-1}\left(\theta^{p}\left(\varepsilon_{1}\right)\right) ; \bar{p}\right) .\right.
\end{aligned}
$$

By adding $\left({ }_{\theta} Y_{\sigma, \tau, \delta, \omega, \theta^{-1}\left(\theta^{p}\left(\varepsilon_{1}\right)\right)_{+}}^{\rho, r, k \circ,} \varphi\right)\left(\theta^{-1}\left(\theta^{p}\left(\varepsilon_{2}\right)\right) ; \bar{p}\right)$ on both sides of (29), we have

$$
\begin{aligned}
& 2\left({ }_{\theta} \Upsilon_{\sigma, \tau, \delta, \omega, \theta^{-1}\left(\theta^{p}\left(\varepsilon_{1}\right)\right)_{+}, r, k, c} \varphi \circ \psi\right)\left(\theta^{-1}\left(\theta^{p}\left(\varepsilon_{2}\right)\right) ; \bar{p}\right) \\
& =\left({ }_{\theta} \Upsilon_{\sigma, \tau, \tau, \delta, \omega, \theta^{-1}\left(\theta^{p}\left(\varepsilon_{1}\right)\right)_{+}}^{\rho, r, c} \varphi \circ \psi\right)\left(\theta^{-1}\left(\theta^{p}\left(\varepsilon_{2}\right)\right) ; \bar{p}\right) \\
& \quad+\left({ }_{\theta}^{\rho, r, k, c}{ }_{\sigma, \tau, \delta, \omega, \theta^{-1}}^{\left.\rho, \theta^{p}\left(\varepsilon_{2}\right)\right)_{-}} \varphi \circ \psi\right)\left(\theta^{-1}\left(\theta^{p}\left(\varepsilon_{1}\right)\right) ; \bar{p}\right) .
\end{aligned}
$$

From equations (29) and (30), the required result can be obtained.

(ii) Proof is on the same lines as the proof of (i).

Theorem 4. Let $\varphi, \theta, h:\left[\varepsilon_{1}, \varepsilon_{2}\right] \subset(0, \infty) \longrightarrow \mathbb{R}$, Range $(\theta)$, Range $(h) \subset\left[\varepsilon_{1}, \varepsilon_{2}\right]$ be the functions such that $\varphi$ be positive and $\varphi \in L_{1}\left(\varepsilon_{1}, \varepsilon_{2}\right), \varepsilon_{1}<\varepsilon_{2}$, and $\theta$ be a differentiable and strictly increasing function where $h$ is a non-negative and integrable function. If $\varphi$ is $p$-convex, $p \in \mathbb{R} \backslash\{0\}$, and $\varphi\left(\theta^{1 / p}(x)\right)=\varphi\left(\left(\theta^{p}\left(\varepsilon_{1}\right)+\theta^{p}\left(\varepsilon_{2}\right)-\theta(x)\right)^{1 / p}\right)$, then the following inequalities for generalized fractional integral operators (5) and (6) hold:

(i) If $p>0$, then

$$
\begin{aligned}
& \varphi\left(\left(\frac{\theta^{p}\left(\varepsilon_{1}\right)+\theta^{p}\left(\varepsilon_{2}\right)}{2}\right)^{1 / p}\right) \\
& \cdot\left(\left({ }_{\theta} \Upsilon_{\sigma, \tau, \delta, \omega^{\prime}, \theta^{-1}\left(\theta^{p}\left(\varepsilon_{1}\right)\right)_{+}}^{\rho, r, k, c} h \circ \psi\right)\left(\theta^{-1}\left(\theta^{p}\left(\varepsilon_{2}\right)\right) ; \bar{p}\right)\right. \\
& \left.+\left({ }_{\theta} \Upsilon_{\sigma, \tau, \delta, \omega^{\prime}, \theta^{-1}\left(\theta^{p}\left(\varepsilon_{2}\right)\right)_{-}^{\rho, r, k, c}} h \circ \psi\right)\left(\theta^{-1}\left(\theta^{p}\left(\varepsilon_{1}\right)\right) ; \bar{p}\right)\right) \\
& \leq\left({ }_{\theta} \Upsilon_{\sigma, \tau, \delta, \omega^{\prime}, \theta^{-1}\left(\theta^{p}\left(\varepsilon_{1}\right)\right)_{+}}^{\rho, r, k, c} \varphi h \circ \psi\right)\left(\theta^{-1}\left(\theta^{p}\left(\varepsilon_{2}\right)\right) ; \bar{p}\right) \\
& +\left({ }_{\theta} \Upsilon_{\sigma, \tau, \delta, \omega^{\prime}, \theta^{-1}\left(\theta^{p}\left(\varepsilon_{2}\right)\right)_{-}, r, k, c} \varphi h \circ \psi\right)\left(\theta^{-1}\left(\theta^{p}\left(\varepsilon_{1}\right)\right) ; \bar{p}\right) \\
& \leq \frac{\varphi\left(\theta\left(\varepsilon_{1}\right)\right)+\varphi\left(\theta\left(\varepsilon_{2}\right)\right)}{2} \\
& \cdot\left(\left({ }_{\theta} \Upsilon_{\sigma, \tau, \delta, \omega^{\prime}, \theta^{-1}\left(\theta^{p}\left(\varepsilon_{1}\right)\right)_{+}}^{\rho, r, k, c} h \circ \psi\right)\left(\theta^{-1}\left(\theta^{p}\left(\varepsilon_{2}\right)\right) ; \bar{p}\right)\right. \\
& \left.+\left({ }_{\theta} \Upsilon_{\sigma, \tau, \delta, \omega^{\prime}, \theta^{-1}\left(\theta^{p}\left(\varepsilon_{2}\right)\right)_{-}}^{\rho, r, k, c} h \circ \psi\right)\left(\theta^{-1}\left(\theta^{p}\left(\varepsilon_{1}\right)\right) ; \bar{p}\right)\right),
\end{aligned}
$$

where $\omega^{\prime}=\omega /\left(\theta^{p}\left(\varepsilon_{2}\right)-\theta^{p}\left(\varepsilon_{1}\right)\right)^{\sigma}$ and $\psi(t)=\theta^{1 / p}(t)$ for all $t \in\left[\varepsilon_{1}^{p}, \varepsilon_{2}^{p}\right]$.

(ii) If $p<0$, then

$$
\begin{aligned}
& \varphi\left(\left(\frac{\theta^{p}\left(\varepsilon_{1}\right)+\theta^{p}\left(\varepsilon_{2}\right)}{2}\right)^{1 / p}\right)\left(\left({ }_{\theta} \Upsilon_{\sigma, \tau, \delta, \omega^{\prime}, \theta^{-1}\left(\theta^{p}\left(\varepsilon_{2}\right)\right)_{+}}^{\rho, r, k, c} h \circ \psi\right)\left(\theta^{-1}\left(\theta^{p}\left(\varepsilon_{1}\right)\right) ; \bar{p}\right)\right. \\
& \left.+\left({ }_{\theta} \Upsilon_{\sigma, \tau, \delta, \omega^{\prime}, \theta^{-1}\left(\theta^{p}\left(\varepsilon_{1}\right)\right)_{-}}^{\rho, r, k, \psi}{ }^{\prime}\right)\left(\theta^{-1}\left(\theta^{p}\left(\varepsilon_{2}\right)\right) ; \bar{p}\right)\right) \\
& \leq\left({ }_{\theta} \Upsilon_{\sigma, \tau, \delta, \omega^{\prime}, \theta^{-1}\left(\theta^{p}\left(\varepsilon_{2}\right)\right)_{+}}^{\rho, r, k, c} \varphi h \circ \psi\right)\left(\theta^{-1}\left(\theta^{p}\left(\varepsilon_{1}\right)\right) ; \bar{p}\right) \\
& +\left({ }_{\theta} \Upsilon_{\sigma, \tau, \delta, \omega^{\prime}, \theta^{-1}\left(\theta^{p}\left(\varepsilon_{1}\right)\right)_{-}}^{\rho, r, k, c} \varphi h \circ \psi\right)\left(\theta^{-1}\left(\theta^{p}\left(\varepsilon_{2}\right)\right) ; \bar{p}\right) \\
& \leq \frac{\varphi\left(\theta\left(\varepsilon_{1}\right)\right)+\varphi\left(\theta\left(\varepsilon_{2}\right)\right)}{2}\left(\left({ }_{\theta} \Upsilon_{\sigma, \tau, \delta, \omega^{\prime}, \theta^{-1}\left(\theta^{p}\left(\varepsilon_{2}\right)\right)_{+}}^{\rho, r, k, c} h \circ \psi\right)\left(\theta^{-1}\left(\theta^{p}\left(\varepsilon_{1}\right)\right) ; \bar{p}\right)+\left({ }_{\theta} \Upsilon_{\sigma, \tau, \delta, \omega^{\prime}, \theta^{-1}\left(\theta^{p}\left(\varepsilon_{1}\right)\right)_{-}^{\rho, r, k, c}}^{h \circ \psi}\right)\left(\theta^{-1}\left(\theta^{p}\left(\varepsilon_{2}\right)\right) ; \bar{p}\right)\right),
\end{aligned}
$$


where $\omega^{\prime}=\omega /\left(\theta^{p}\left(\varepsilon_{1}\right)-\theta^{p}\left(\varepsilon_{2}\right)\right)^{\sigma}$ and $\psi(t)=\theta^{1 / p}(t)$ for all $t \in\left[\varepsilon_{2}^{p}, \varepsilon_{1}^{p}\right]$.
Proof. (i) Multiplying both sides of (17) by $2 t^{\tau-1} E_{\sigma, \tau, \delta}^{\rho, r, k, c}\left(\omega t^{\sigma} ; \bar{p}\right) h\left(\left(t \theta^{p}\left(\varepsilon_{1}\right)+(1-t) \theta^{p}\left(\varepsilon_{2}\right)\right)^{1 / p}\right)$ and then integrating over $[0,1]$, we have

$$
\begin{aligned}
& 2 \varphi\left(\left(\frac{\theta^{p}\left(\varepsilon_{1}\right)+\theta^{p}\left(\varepsilon_{2}\right)}{2}\right)^{1 / p}\right) \int_{0}^{1} t^{\tau-1} E_{\sigma, \tau, \delta}^{\rho, r, k, c}\left(\omega t^{\sigma} ; \bar{p}\right) h\left(\left(t \theta^{p}\left(\varepsilon_{1}\right)+(1-t) \theta^{p}\left(\varepsilon_{2}\right)\right)^{1 / p}\right) \mathrm{d} t \\
& \leq \int_{0}^{1} t^{\tau-1} E_{\sigma, \tau, \delta}^{\rho, r, k, c}\left(\omega t^{\sigma} ; \bar{p}\right) \varphi\left(\left(t \theta^{p}\left(\varepsilon_{1}\right)+(1-t) \theta^{p}\left(\varepsilon_{2}\right)\right)^{1 / p}\right) h\left(\left(t \theta^{p}\left(\varepsilon_{1}\right)+(1-t) \theta^{p}\left(\varepsilon_{2}\right)\right)^{1 / p}\right) \mathrm{d} t \\
& \quad+\int_{0}^{1} t^{\tau-1} E_{\sigma, \tau, \delta}^{\rho, r, k, c}\left(\omega t^{\sigma} ; \bar{p}\right) \varphi\left(\left(t \theta^{p}\left(\varepsilon_{2}\right)+(1-t) \theta^{p}\left(\varepsilon_{1}\right)\right)^{1 / p}\right) h\left(\left(t \theta^{p}\left(\varepsilon_{1}\right)+(1-t) \theta^{p}\left(\varepsilon_{2}\right)\right)^{1 / p}\right) \mathrm{d} t .
\end{aligned}
$$

By choosing $\theta(x)=t \theta^{p}\left(\varepsilon_{1}\right)+(1-t) \theta^{p}\left(\varepsilon_{2}\right)$, that is, $\theta^{p}\left(\varepsilon_{1}\right)+\theta^{p}\left(\varepsilon_{2}\right)-\theta(x)=t \theta^{p}\left(\varepsilon_{2}\right)+(1-t) \theta^{p}\left(\varepsilon_{1}\right), \quad$ in $\quad(33)$

and using $\varphi\left(\theta^{1 / p}(x)\right)=\varphi\left(\left(\theta^{p}\left(\varepsilon_{1}\right)+\theta^{p}\left(\varepsilon_{2}\right)-\theta(x)\right)^{1 / p}\right)$, we have

$$
\begin{aligned}
& 2 \varphi\left(\left(\frac{\theta^{p}\left(\varepsilon_{1}\right)+\theta^{p}\left(\varepsilon_{2}\right)}{2}\right)^{1 / p}\right) \\
& \quad \cdot \int_{\theta^{-1}\left(\theta^{p}\left(\varepsilon_{1}\right)\right)}^{\theta^{-1}\left(\theta^{p}\left(\varepsilon_{2}\right)\right)}\left(\theta^{p}\left(\varepsilon_{2}\right)-\theta(x)\right)^{\tau-1} E_{\sigma, \tau, \delta}^{\rho, r, k, c}\left(\omega^{\prime}\left(\theta^{p}\left(\varepsilon_{2}\right)-\theta(x)\right)^{\sigma} ; \bar{p}\right) h \circ \psi(x) \mathrm{d}(\theta(x)) \\
& \leq \int_{\theta^{-1}\left(\theta^{p}\left(\varepsilon_{1}\right)\right)}^{\theta^{-1}\left(\theta^{p}\left(\varepsilon_{2}\right)\right)}\left(\theta^{p}\left(\varepsilon_{2}\right)-\theta(x)\right)^{\tau-1} E_{\sigma, \tau, \delta}^{\rho, r, k, c}\left(\omega^{\prime}\left(\theta^{p}\left(\varepsilon_{2}\right)-\theta(x)\right)^{\sigma} ; \bar{p}\right) \varphi h \circ \psi(x) \mathrm{d}(\theta(x)) \\
& \quad+\int_{\theta^{-1}\left(\theta^{p}\left(\varepsilon_{1}\right)\right)}^{\theta^{-1}\left(\theta^{p}\left(\varepsilon_{2}\right)\right)}\left(\theta(x)-\theta^{p}\left(\varepsilon_{1}\right)\right)^{\tau-1} E_{\sigma, \tau, \delta}^{\rho, r, k, c}\left(\omega^{\prime}\left(\theta(x)-\theta^{p}\left(\varepsilon_{1}\right)\right)^{\sigma} ; \bar{p}\right) \varphi h \circ \psi(x) \mathrm{d}(\theta(x)) .
\end{aligned}
$$

This implies

$$
\begin{aligned}
& 2 \varphi\left(\left(\frac{\theta^{p}\left(\varepsilon_{1}\right)+\theta^{p}\left(\varepsilon_{2}\right)}{2}\right)^{1 / p}\right)\left({ }_{\theta} \Upsilon_{\sigma, \tau, \delta, \omega^{\prime}, \theta^{-1}\left(\theta^{p}\left(\varepsilon_{1}\right)\right)_{+}}^{\rho, r, c} h \circ \psi\right)\left(\theta^{-1}\left(\theta^{p}\left(\varepsilon_{2}\right)\right) ; \bar{p}\right) \\
& \leq\left({ }_{\theta} \Upsilon_{\sigma, \tau, \delta, \omega^{\prime}, \theta^{-1}\left(\theta^{p}\left(\varepsilon_{1}\right)\right)_{+}, r, c} \varphi h \circ \psi\right)\left(\theta^{-1}\left(\theta^{p}\left(\varepsilon_{2}\right)\right) ; \bar{p}\right) \\
& +\left({ }_{\theta} Y_{\sigma, \tau, \delta, \omega^{\prime}, \theta^{-1}\left(\theta^{p}\left(\varepsilon_{2}\right)\right)_{-}}^{\rho, r, c} \varphi h \circ \psi\right)\left(\theta^{-1}\left(\theta^{p}\left(\varepsilon_{1}\right)\right) ; \bar{p}\right) .
\end{aligned}
$$

Using Lemma 1 (i) in above inequality, we have 


$$
\begin{aligned}
& \varphi\left(\left(\frac{\theta^{p}\left(\varepsilon_{1}\right)+\theta^{P}\left(\varepsilon_{2}\right)}{2}\right)^{1 / p}\right)\left(\left({ }_{\theta} \Upsilon_{\sigma, \tau, \delta, \omega^{\prime}, \theta^{-1}\left(\theta^{p}\left(\varepsilon_{1}\right)\right)_{+}}^{\rho, r, k \circ \psi}\right)\left(\theta^{-1}\left(\theta^{p}\left(\varepsilon_{2}\right)\right) ; \bar{p}\right)\right. \\
& \left.+\left({ }_{\theta} \Upsilon_{\sigma, \tau, \delta, \omega^{\prime}, \theta^{-1}\left(\theta^{p}\left(\varepsilon_{2}\right)\right)_{-}}^{\rho, r, k, c} h \circ \psi\right)\left(\theta^{-1}\left(\theta^{p}\left(\varepsilon_{1}\right)\right) ; \bar{p}\right)\right) \\
& \leq\left({ }_{\theta} Y_{\sigma, \tau, \delta, \omega^{\prime}, \theta^{-1}\left(\theta^{p}\left(\varepsilon_{1}\right)\right)_{+}}^{\rho, r, k, c} \varphi h \circ \psi\right)\left(\theta^{-1}\left(\theta^{p}\left(\varepsilon_{2}\right)\right) ; \bar{p}\right) \\
& +\left({ }_{\theta} \Upsilon_{\sigma, \tau, \delta, \omega^{\prime}, \theta^{-1}\left(\theta^{p}\left(\varepsilon_{2}\right)\right)_{-}, r, k, c} \varphi h \circ \psi\right)\left(\theta^{-1}\left(\theta^{p}\left(\varepsilon_{1}\right)\right) ; \bar{p}\right) .
\end{aligned}
$$

To prove the second inequality of (31), multiplying both sides of $(21)$ by $t^{\tau-1} E_{\sigma, \tau, \delta}^{\rho, r, k, c}\left(\omega t^{\sigma} ; \bar{p}\right) h\left(\left(t \theta^{p}\left(\varepsilon_{1}\right)+(1-t) \theta^{p}\right.\right.$ $\left.\left(\varepsilon_{2}\right)\right)^{1 / p}$ ) and then integrating over $[0,1]$, we have

$$
\begin{aligned}
& \int_{0}^{1} t^{\tau-1} E_{\sigma, \tau, \delta}^{\rho, r, k, c}\left(\omega t^{\sigma} ; \bar{p}\right) \varphi\left(\left(t \theta^{p}\left(\varepsilon_{1}\right)+(1-t) \theta^{p}\left(\varepsilon_{2}\right)\right)^{1 / p}\right) h\left(\left(t \theta^{p}\left(\varepsilon_{1}\right)+(1-t) \theta^{p}\left(\varepsilon_{2}\right)\right)^{1 / p}\right) \mathrm{d} t \\
& \quad+\int_{0}^{1} t^{\tau-1} E_{\sigma, \tau, \delta}^{\rho, r, k, c}\left(\omega t^{\sigma} ; \bar{p}\right) \varphi\left(\left(t \theta^{p}\left(\varepsilon_{2}\right)+(1-t) \theta^{p}\left(\varepsilon_{1}\right)\right)^{1 / p}\right) h\left(\left(t \theta^{p}\left(\varepsilon_{1}\right)+(1-t) \theta^{p}\left(\varepsilon_{2}\right)\right)^{1 / p}\right) \mathrm{d} t \\
& \leq\left(\varphi\left(\theta\left(\varepsilon_{1}\right)\right)+\varphi\left(\theta\left(\varepsilon_{2}\right)\right)\right) \int_{0}^{1} t^{\tau-1} E_{\sigma, \tau, \delta}^{\rho, r, k, c}\left(\omega t^{\sigma} ; \bar{p}\right) h\left(\left(t \theta^{p}\left(\varepsilon_{1}\right)+(1-t) \theta^{p}\left(\varepsilon_{2}\right)\right)^{1 / p}\right) \mathrm{d} t .
\end{aligned}
$$

Setting $\theta(x)=t \theta^{P}\left(\varepsilon_{1}\right)+(1-t) \theta^{P}\left(\varepsilon_{2}\right)$ and using $\varphi\left(\theta^{1 / p}(x)\right)=\varphi\left(\left(\theta^{p}\left(\varepsilon_{1}\right)+\theta^{p}\left(\varepsilon_{2}\right)-\theta(x)\right)^{1 / p}\right)$ in (37) and after simplification, we have

$$
\begin{aligned}
& \left({ }_{\theta} \mathrm{Y}_{\sigma, \tau, \delta, \omega^{\prime}, \theta^{-1}\left(\theta^{p}\left(\varepsilon_{1}\right)\right)_{+}}^{\rho, k, c} \varphi h \circ \psi\right)\left(\theta^{-1}\left(\theta^{p}\left(\varepsilon_{2}\right)\right) ; \bar{p}\right) \\
& +\left({ }_{\theta} \Upsilon_{\sigma, \tau, \delta, \omega^{\prime}, \theta^{-1}}^{\left.\rho, \theta^{p}\left(\varepsilon_{2}\right)\right)_{-}} \varphi h \circ \psi\right)\left(\theta^{-1}\left(\theta^{p}\left(\varepsilon_{1}\right)\right) ; \bar{p}\right) \\
& \leq\left(\varphi\left(\theta\left(\varepsilon_{1}\right)\right)+\varphi\left(\theta\left(\varepsilon_{2}\right)\right)\right)\left({ }_{\theta} \Upsilon_{\sigma, \tau, \delta, \omega^{\prime}, \theta^{-1}\left(\theta^{p}\left(\varepsilon_{1}\right)\right)_{+}}^{\rho, r, c}{ }^{\circ} \%\right)\left(\theta^{-1}\left(\theta^{p}\left(\varepsilon_{2}\right)\right) ; \bar{p}\right) \text {. }
\end{aligned}
$$

Using Lemma 1 (i), inequality (38) becomes

$$
\begin{aligned}
& \left({ }_{\theta} \Upsilon_{\sigma, \tau, \delta, \omega^{\prime}, \theta^{-1}\left(\theta^{p}\left(\varepsilon_{1}\right)\right)_{+}, r, k} \varphi h \circ \psi\right)\left(\theta^{-1}\left(\theta^{p}\left(\varepsilon_{2}\right)\right) ; \bar{p}\right) \\
& +\left({ }_{\theta} \Upsilon_{\sigma, \tau, \delta, \omega^{\prime}, \theta^{-1}\left(\theta^{p}\left(\varepsilon_{2}\right)\right)_{-}}^{\rho, r, k, c} \varphi h \circ \psi\right)\left(\theta^{-1}\left(\theta^{p}\left(\varepsilon_{1}\right)\right) ; \bar{p}\right) \\
& \leq \frac{\left(\varphi\left(\theta\left(\varepsilon_{1}\right)\right)+\varphi\left(\theta\left(\varepsilon_{2}\right)\right)\right)}{2} \\
& \cdot\left(\left({ }_{\theta} \Upsilon_{\sigma, \tau, \delta, \omega^{\prime}, \theta^{-1}\left(\theta^{p}\left(\varepsilon_{1}\right)\right)_{+}}^{\rho, r, k}{ }^{h} \psi \psi\right)\left(\theta^{-1}\left(\theta^{p}\left(\varepsilon_{2}\right)\right) ; \bar{p}\right)\right. \\
& \left.+\left({ }_{\theta} \Upsilon_{\sigma, \tau, \delta, \omega^{\prime}, \theta^{-1}\left(\theta^{p}\left(\varepsilon_{2}\right)\right)_{-}}^{\rho, r, k, c} h \circ \psi\right)\left(\theta^{-1}\left(\theta^{p}\left(\varepsilon_{1}\right)\right) ; \bar{p}\right)\right) .
\end{aligned}
$$

By combining (36) and (39), we get (31).

(ii) Proof is similar to the proof of (i) by using (ii) of Lemma 1.
Remark 3

(i) By setting $\bar{p}=0$ and $\theta=I$, Theorem 2.2 of [25] is obtained.

(ii) By setting $\bar{p}=\omega=0$ and $\theta=I$, Theorem 9 of [11] is obtained.

(iii) By setting $\bar{p}=0, h(x)=1, p=-1$, and $\theta=I$, Theorem 2.1 of [22] is obtained.

(iv) By setting $\theta=I, h(x)=1$, and $p=-1$, Theorem 2.1 of [23] is obtained.

(v) By setting $\omega=\bar{p}=0, h(x)=1, p=-1$, and $\theta=I$, Theorem 4 of [18] is obtained.

(vi) By setting $p=-1$, Theorem 2.5 of [24] is obtained.

Corollary 2. If we put $p=-1, \bar{p}=0$, and $\theta=I$ in Theorem 4 (ii), we get the following Fejér-Hadamard inequalities for harmonically convex function via generalized fractional integral operators: 


$$
\begin{aligned}
& \varphi\left(\frac{2 \varepsilon_{1} \varepsilon_{2}}{\varepsilon_{1}+\varepsilon_{2}}\right)\left(\left({ }_{\theta} \Upsilon_{\sigma, \tau, \delta, \omega^{\prime},\left(1 / \varepsilon_{2}\right)^{+}}^{\rho, r, k, c} h \circ \psi\right)\left(\frac{1}{\varepsilon_{1}}\right)+\left({ }_{\theta} \Upsilon_{\sigma, \tau, \delta, \omega^{\prime},\left(1 / \varepsilon_{1}\right)^{-, r, k, c}}^{\rho} h \circ \psi\right)\left(\frac{1}{\varepsilon_{2}}\right)\right) \\
& \leq\left({ }_{\theta} \Upsilon_{\sigma, \tau, \delta, \omega^{\prime},\left(1 / \varepsilon_{2}\right)^{+}}^{\rho, r, k, c} \varphi h \circ \psi\right)\left(\frac{1}{\varepsilon_{1}}\right)+\left({ }_{\theta} \Upsilon_{\sigma, \tau, \delta, \omega^{\prime},\left(1 / \varepsilon_{1}\right)^{-}}^{\rho, r, k, c} \varphi h \circ \psi\right)\left(\frac{1}{\varepsilon_{2}}\right) \\
& \leq \frac{\varphi\left(\varepsilon_{1}\right)+\varphi\left(\varepsilon_{2}\right)}{2}\left(\left({ }_{\theta} \Upsilon_{\sigma, \tau, \delta, \omega^{\prime},\left(1 / \varepsilon_{2}\right)^{+}}^{\rho, r, k, c} h \circ \psi\right)\left(\frac{1}{\varepsilon_{1}}\right)+\left({ }_{\theta} \Upsilon_{\sigma, \tau, \delta, \omega^{\prime},\left(1 / \varepsilon_{1}\right)^{-}}^{\rho, r, k, c} h \circ \psi\right)\left(\frac{1}{\varepsilon_{2}}\right)\right) .
\end{aligned}
$$

Now we give another version of the Hadamard inequality.

Theorem 5. Let $\varphi, \theta:\left[\varepsilon_{1}, \varepsilon_{2}\right] \subset(0, \infty) \longrightarrow \mathbb{R}$, Range $(\theta) \subset\left[\varepsilon_{1}, \varepsilon_{2}\right]$ be the functions such that $\varphi$ be positive and $\varphi \in L_{1}\left[\varepsilon_{1}, \varepsilon_{2}\right], \varepsilon_{1}<\varepsilon_{2}$, and $\theta$ be a differentiable and strictly increasing function. If $\varphi$ is $p$-convex, $p \in \mathbb{R} \backslash\{0\}$, then for generalized fractional integral operators (5) and (6), we have

(i) If $p>0$, then

$$
\begin{aligned}
& \varphi\left(\left(\frac{\theta^{p}\left(\varepsilon_{1}\right)+\theta^{p}\left(\varepsilon_{2}\right)}{2}\right)^{1 / p}\right)\left({ }_{\theta} Y_{\sigma, \tau, \delta, 2^{\sigma} \omega^{\prime}, \theta^{-1}\left(\left(\theta^{p}\left(\varepsilon_{1}\right)+\theta^{p}\left(\varepsilon_{2}\right)\right) / 2\right)_{+}}^{\rho, r, k}\right)\left(\theta^{-1}\left(\theta^{p}\left(\varepsilon_{2}\right)\right) ; \bar{p}\right) \\
& \leq\left({ }_{\theta} Y_{\sigma, \tau, \delta, 2^{\sigma} \omega^{\prime}, \theta^{-1}}^{\left.\rho, r, k\left(\theta^{p}\left(\varepsilon_{1}\right)+\theta^{p}\left(\varepsilon_{2}\right)\right) / 2\right)_{+}} \varphi^{\circ} \psi\right)\left(\theta^{-1}\left(\theta^{p}\left(\varepsilon_{2}\right)\right) ; \bar{p}\right) \\
& +\left({ }_{\theta} \Upsilon_{\sigma, \tau, \delta, 2^{\sigma} \omega^{\prime}, \theta^{-1}\left(\left(\theta^{p}\left(\varepsilon_{1}\right)+\theta^{p}\left(\varepsilon_{2}\right)\right) / 2\right)_{-}}^{\rho, r, k, \mathcal{L}} \varphi\right)\left(\theta^{-1}\left(\theta^{p}\left(\varepsilon_{1}\right)\right) ; \bar{p}\right) \\
& \leq \frac{\varphi\left(\theta\left(\varepsilon_{1}\right)\right)+\varphi\left(\theta\left(\varepsilon_{2}\right)\right)}{2}\left({ }_{\theta} \Upsilon_{\sigma, \tau, \delta, 2^{\sigma} \omega^{\prime}, \theta^{-1}}^{\rho, r, k, c}\left(\left(\theta^{p}\left(\varepsilon_{1}\right)+\theta^{p}\left(\varepsilon_{2}\right)\right) / 2\right)_{+}{ }^{1}\right)\left(\theta^{-1}\left(\theta^{p}\left(\varepsilon_{2}\right)\right) ; \bar{p}\right),
\end{aligned}
$$

where $\omega^{\prime}=\omega /\left(\theta^{p}\left(\varepsilon_{2}\right)-\theta^{p}\left(\varepsilon_{1}\right)\right)^{\sigma}$ and $\psi(t)=\theta^{1 / p}(t)$ for all $t \in\left[\varepsilon_{1}^{p}, \varepsilon_{2}^{p}\right]$.

(ii) If $p<0$, then

$$
\begin{aligned}
& \varphi\left(\left(\frac{\theta^{p}\left(\varepsilon_{1}\right)+\theta^{p}\left(\varepsilon_{2}\right)}{2}\right)^{1 / p}\right)\left({ }_{\theta} \Upsilon_{\sigma, \tau, \delta, 2^{\sigma} \omega^{\prime}, \theta^{-1}\left(\left(\theta^{p}\left(\varepsilon_{1}\right)+\theta^{p}\left(\varepsilon_{2}\right)\right) / 2\right)_{-}}^{1, r, k}\right)\left(\theta^{-1}\left(\theta^{p}\left(\varepsilon_{2}\right)\right) ; \bar{p}\right) \\
& \left.\leq\left({ }_{\theta} \Upsilon_{\sigma, \tau, \delta, 2^{\sigma} \omega^{\prime}, \theta^{-1}}^{\rho, r, k,(}\left(\theta^{p}\left(\varepsilon_{1}\right)+\theta^{p}\left(\varepsilon_{2}\right)\right) / 2\right)_{+}{ }^{\circ \circ \psi}\right)\left(\theta^{-1}\left(\theta^{p}\left(\varepsilon_{1}\right)\right) ; \bar{p}\right) \\
& +\left({ }_{\theta} \Upsilon_{\sigma, \tau, \delta, 2^{\sigma} \omega^{\prime}, \theta^{-1}\left(\left(\theta^{p}\left(\varepsilon_{1}\right)+\theta^{p}\left(\varepsilon_{2}\right)\right) / 2\right)_{-}}^{\rho, r, k, c} \varphi \%\right)\left(\theta^{-1}\left(\theta^{p}\left(\varepsilon_{2}\right)\right) ; \bar{p}\right) \\
& \leq \frac{\varphi\left(\theta\left(\varepsilon_{1}\right)\right)+\varphi\left(\theta\left(\varepsilon_{2}\right)\right)}{2}\left({ }_{\theta} \Upsilon_{\sigma, \tau, \delta, 2^{\sigma} \omega^{\prime}, \theta^{-1}}^{\rho, r, k, c}\left(\left(\theta^{p}\left(\varepsilon_{1}\right)+\theta^{p}\left(\varepsilon_{2}\right)\right) / 2\right)_{-} 1\right)\left(\theta^{-1}\left(\theta^{p}\left(\varepsilon_{2}\right)\right) ; \bar{p}\right),
\end{aligned}
$$

where $\omega^{\prime}=\omega /\left(\theta^{p}\left(\varepsilon_{1}\right)-\theta^{p}\left(\varepsilon_{2}\right)\right)^{\sigma}$ and $\psi(t)=\theta^{1 / p}(t)$ for all $t \in\left[\varepsilon_{2}^{p}, \varepsilon_{1}^{p}\right]$.
Proof. (i) Setting $\theta(x)=\left((t / 2) \theta^{p}\left(\varepsilon_{1}\right)+((2-t) / 2) \theta^{P}\left(\varepsilon_{2}\right)\right)^{1 / p}$ and $\left.\theta(y)=\left((t / 2) \theta^{p}\left(\varepsilon_{2}\right)+((2-t) / 2)\right) \theta^{p}\left(\varepsilon_{1}\right)\right)^{1 / p}$ in (12), we have 


$$
\begin{aligned}
& \varphi\left(\left(\frac{\theta^{p}\left(\varepsilon_{1}\right)+\theta^{p}\left(\varepsilon_{2}\right)}{2}\right)^{1 / p}\right) \\
& \leq \frac{\varphi\left(\left((t / 2) \theta^{p}\left(\varepsilon_{1}\right)+((2-t) / 2) \theta^{p}\left(\varepsilon_{2}\right)\right)^{1 / p}\right)+\varphi\left(\left((t / 2) \theta^{p}\left(\varepsilon_{2}\right)+((2-t) / 2) \theta^{p}\left(\varepsilon_{1}\right)\right)^{1 / p}\right)}{2} .
\end{aligned}
$$

Multiplying both sides of $(43)$ by $2 t^{\tau-1} E_{\sigma, \tau, \delta}^{\rho, r, c}\left(\omega t^{\sigma} ; \bar{p}\right)$ and then integrating over $[0,1]$, we have

$$
\begin{aligned}
& 2 \varphi\left(\left(\frac{\theta^{p}\left(\varepsilon_{1}\right)+\theta^{p}\left(\varepsilon_{2}\right)}{2}\right)^{1 / p}\right) \int_{0}^{1} t^{\tau-1} E_{\sigma, \tau, \delta}^{\rho, r, k, c}\left(\omega t^{\sigma} ; \bar{p}\right) \mathrm{d} t \\
& \leq \int_{0}^{1} t^{\tau-1} E_{\sigma, \tau, \delta}^{\rho, r, k, c}\left(\omega t^{\sigma} ; \bar{p}\right) \varphi\left(\left(\frac{t}{2} \theta^{p}\left(\varepsilon_{1}\right)+((2-t) / 2) \theta^{p}\left(\varepsilon_{2}\right)\right)^{1 / p}\right) \mathrm{d} t \\
& \quad+\int_{0}^{1} t^{\tau-1} E_{\sigma, \tau, \delta}^{\rho, r, k, c}\left(\omega t^{\sigma} ; \bar{p}\right) \varphi\left(\left(\frac{t}{2} \theta^{p}\left(\varepsilon_{2}\right)+((2-t) / 2) \theta^{p}\left(\varepsilon_{1}\right)\right)^{1 / p}\right) \mathrm{d} t .
\end{aligned}
$$

By choosing $\theta(x)=(t / 2) \theta^{p}\left(\varepsilon_{1}\right)+((2-t) / 2) \theta^{p}\left(\varepsilon_{2}\right)$ and $\theta(y)=(t / 2) \theta^{p}\left(\varepsilon_{2}\right)+((2-t) / 2) \theta^{p}\left(\varepsilon_{1}\right)$ in (44) and by (5) and (6), we get first inequality of (41).

To prove the second inequality of (41), again from $p$-convexity of $\varphi$ over $\left[\varepsilon_{1}, \varepsilon_{2}\right]$ and for $t \in[0,1]$, we have

$$
\begin{aligned}
& \varphi\left(\left(\frac{t}{2} \theta^{p}\left(\varepsilon_{1}\right)+\left(\frac{2-t}{2}\right) \theta^{p}\left(\varepsilon_{2}\right)\right)^{1 / p}\right) \\
& \quad+\varphi\left(\left(\frac{t}{2} \theta^{p}\left(\varepsilon_{2}\right)+\left(\frac{2-t}{2}\right) \theta^{p}\left(\varepsilon_{1}\right)\right)^{1 / p}\right) \\
& \leq \varphi\left(\theta\left(\varepsilon_{1}\right)\right)+\varphi\left(\theta\left(\varepsilon_{2}\right)\right) .
\end{aligned}
$$

Multiplying both sides of $(45)$ by $t^{\tau-1} E_{\sigma, \tau, \delta}^{\rho, r, k, c}\left(\omega t^{\sigma} ; \bar{p}\right)$ and then integrating over $[0,1]$, we have

$$
\begin{aligned}
& \int_{0}^{1} t^{\tau-1} E_{\sigma, \tau, \delta}^{\rho, r, c}\left(\omega t^{\sigma} ; \bar{p}\right) \varphi\left(\left(\frac{t}{2} \theta^{p}\left(\varepsilon_{1}\right)+\left(\frac{2-t}{2}\right) \theta^{p}\left(\varepsilon_{2}\right)\right)^{1 / p}\right) \mathrm{d} t \\
& \quad+\int_{0}^{1} t^{\tau-1} E_{\sigma, \tau, \delta}^{\rho, r, k, c}\left(\omega t^{\sigma} ; \bar{p}\right) \varphi\left(\left(\frac{t}{2} \theta^{p}\left(\varepsilon_{2}\right)+\left(\frac{2-t}{2}\right) \theta^{p}\left(\varepsilon_{1}\right)\right)^{1 / p}\right) \mathrm{d} t \\
& \leq\left(\varphi\left(\theta\left(\varepsilon_{1}\right)\right)+\varphi\left(\theta\left(\varepsilon_{2}\right)\right)\right) \int_{0}^{1} t^{\tau-1} E_{\sigma, \tau, \delta}^{\rho, r, k, c}\left(\omega t^{\sigma} ; \bar{p}\right) \mathrm{d} t .
\end{aligned}
$$

Setting $\theta(x)=(t / 2) \theta^{P}\left(\varepsilon_{1}\right)+((2-t) / 2) \theta^{P}\left(\varepsilon_{2}\right) \quad$ and $\theta(y)=(t / 2) \theta^{P}\left(\varepsilon_{2}\right)+((2-t) / 2) \theta^{P}\left(\varepsilon_{1}\right)$ in (46) and using (5) and (6), we get second inequality of (41).

(ii) Proof is similar to the proof of (i).

Remark 4

(i) By setting $\bar{p}=0, p=-1$, and $\theta=I$, Theorem 2.3 of [22] is obtained.

(ii) By setting $\theta=I$ and $p=-1$, Theorem 2.3 of [23] is obtained.

(iii) By setting $p=-1$, Theorem 2.7 of [24] is obtained.

Now we obtain another Fejér-Hadamard type fractional integral inequality for $p$-convex function via generalized fractional integral operators (5) and (6).

Theorem 6. Let $\varphi, \theta, h:\left[\varepsilon_{1}, \varepsilon_{2}\right] \subset(0, \infty) \longrightarrow \mathbb{R}$, Range $(\theta)$, Range $(h) \subset\left[\varepsilon_{1}, \varepsilon_{2}\right]$ be the functions such that $\varphi$ be positive and $\varphi \in L_{1}\left(\varepsilon_{1}, \varepsilon_{2}\right), \varepsilon_{1}<\varepsilon_{2}$, and $\theta$ be a differentiable and strictly increasing function where $h$ is a non-negative and integrable function. If $\varphi$ is $p$-convex, $p \in \mathbb{R} \backslash\{0\}$, and $\varphi\left(\theta^{1 / p}(x)\right)=\varphi\left(\left(\theta^{p}\left(\varepsilon_{1}\right)+\theta^{p}\left(\varepsilon_{2}\right)-\theta(x)\right)^{1 / p}\right)$, then the following inequalities for generalized fractional integral operators (5) and (6) hold:

(i) If $p>0$, then 


$$
\begin{aligned}
& \varphi\left(\left(\frac{\theta^{p}\left(\varepsilon_{1}\right)+\theta^{p}\left(\varepsilon_{2}\right)}{2}\right)^{1 / p}\right)\left({ }_{\theta} Y_{\sigma, \tau, \delta, 2^{\sigma} \omega^{\prime}, \theta^{-1}\left(\left(\theta^{p}\left(\varepsilon_{1}\right)+\theta^{p}\left(\varepsilon_{2}\right)\right) / 2\right)_{+}}^{\rho, k} h^{\prime} \psi\right)\left(\theta^{-1}\left(\theta^{p}\left(\varepsilon_{2}\right)\right) ; \bar{p}\right)
\end{aligned}
$$

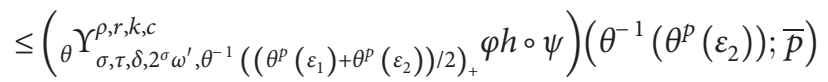

$$
\begin{aligned}
& +\left({ }_{\theta} \mathrm{Y}_{\sigma, \tau, \delta, 2^{\sigma} \omega^{\prime}, \theta^{-1}}^{\rho, r, k, c}\left(\left(\theta^{p}\left(\varepsilon_{1}\right)+\theta^{p}\left(\varepsilon_{2}\right)\right) / 2\right)_{-} \varphi h \circ \psi\right)\left(\theta^{-1}\left(\theta^{p}\left(\varepsilon_{1}\right)\right) ; \bar{p}\right) \\
& \leq \frac{\varphi\left(\theta\left(\varepsilon_{1}\right)\right)+\varphi\left(\theta\left(\varepsilon_{2}\right)\right)}{2}\left({ }_{\theta} Y_{\sigma, \tau, \delta, 2^{\sigma} \omega^{\prime}, \theta^{-1}}^{\left.\left.\rho, r, k, \theta^{p}\left(\varepsilon_{1}\right)+\theta^{p}\left(\varepsilon_{2}\right)\right) / 2\right)_{+}}{ }^{\prime} \circ \psi\right)\left(\theta^{-1}\left(\theta^{p}\left(\varepsilon_{2}\right)\right) ; \bar{p}\right),
\end{aligned}
$$

where $\omega^{\prime}=\omega /\left(\theta^{p}\left(\varepsilon_{2}\right)-\theta^{p}\left(\varepsilon_{1}\right)\right)^{\sigma}$ and $\psi(t)=\theta^{1 / p}(t)$

for all $t \in\left[\varepsilon_{1}^{p}, \varepsilon_{2}^{p}\right]$.

(ii) If $p<0$, then

$$
\begin{aligned}
& \varphi\left(\left(\frac{\theta^{p}\left(\varepsilon_{1}\right)+\theta^{p}\left(\varepsilon_{2}\right)}{2}\right)^{1 / p}\right)\left({ }_{\theta} \Upsilon_{\sigma, \tau, \delta, 2^{\sigma} \omega^{\prime}, \theta^{-1}\left(\left(\theta^{p}\left(\varepsilon_{1}\right)+\theta^{p}\left(\varepsilon_{2}\right)\right) / 2\right)_{-}}^{\rho, k, \psi}\right)\left(\theta^{-1}\left(\theta^{p}\left(\varepsilon_{2}\right)\right) ; \bar{p}\right) \\
& \leq\left({ }_{\theta} \Upsilon_{\sigma, \tau, \delta, 2^{\sigma} \omega^{\prime}, \theta^{-1}}^{\rho, r, c}\left(\left(\theta^{p}\left(\varepsilon_{1}\right)+\theta^{p}\left(\varepsilon_{2}\right)\right) / 2\right)_{+}{ }_{+} \circ h \circ \psi\right)\left(\theta^{-1}\left(\theta^{p}\left(\varepsilon_{1}\right)\right) ; \bar{p}\right) \\
& +\left({ }_{\theta} Y_{\sigma, \tau, \delta, 2^{\sigma} \omega^{\prime}, \theta^{-1}}^{\rho, r, k, c}\left(\left(\theta^{p}\left(\varepsilon_{1}\right)+\theta^{p}\left(\varepsilon_{2}\right)\right) / 2\right)_{-} \varphi h \circ \psi\right)\left(\theta^{-1}\left(\theta^{p}\left(\varepsilon_{2}\right)\right) ; \bar{p}\right) \\
& \left.\leq \frac{\varphi\left(\theta\left(\varepsilon_{1}\right)\right)+\varphi\left(\theta\left(\varepsilon_{2}\right)\right)}{2}\left({ }_{\theta} \Upsilon_{\sigma, \tau, \delta, 2^{\sigma} \omega^{\prime}, \theta^{-1}}^{\rho, r, k, c}\left(\theta^{p}\left(\varepsilon_{1}\right)+\theta^{p}\left(\varepsilon_{2}\right)\right) / 2\right)_{-} h \circ \psi\right)\left(\theta^{-1}\left(\theta^{p}\left(\varepsilon_{2}\right)\right) ; \bar{p}\right),
\end{aligned}
$$

where $\omega^{\prime}=\omega /\left(\theta^{p}\left(\varepsilon_{1}\right)-\theta^{p}\left(\varepsilon_{2}\right)\right)^{\sigma}$ and $\psi(t)=\theta^{1 / p}(t)$ for all $t \in\left[\varepsilon_{2}^{p}, \varepsilon_{1}^{p}\right]$.
Multiplying (43) by $2 t^{\tau-1} E_{\sigma, \tau, \delta}^{\rho, r, k, c}\left(\omega t^{\sigma} ; \bar{p}\right) h\left(\left((t / 2) \theta^{p}\left(\varepsilon_{1}\right)+\right.\right.$ $\left.\left.((2-t) / 2) \theta^{p}\left(\varepsilon_{2}\right)\right)^{1 / p}\right)$ and then integrating over $[0,1]$, we have

Proof. (i)

$$
\begin{aligned}
& 2 \varphi\left(\left(\frac{\theta^{p}\left(\varepsilon_{1}\right)+\theta^{p}\left(\varepsilon_{2}\right)}{2}\right)^{1 / p}\right) \\
& \quad \cdot \int_{0}^{1} t^{\tau-1} E_{\sigma, \tau, \delta}^{\rho, r, k, c}\left(\omega t^{\sigma} ; \bar{p}\right) h\left(\left(\frac{t}{2} \theta^{p}\left(\varepsilon_{1}\right)+\left(\frac{2-t}{2}\right) \theta^{p}\left(\varepsilon_{2}\right)\right)^{1 / p}\right) \mathrm{d} t \\
& \leq \int_{0}^{1} t^{\tau-1} E_{\sigma, \tau, \delta}^{\rho, r, k, c}\left(\omega t^{\sigma} ; \bar{p}\right) \varphi\left(\left(\frac{t}{2} \theta^{p}\left(\varepsilon_{1}\right)+\left(\frac{2-t}{2}\right) \theta^{p}\left(\varepsilon_{2}\right)\right)^{1 / p}\right) h\left(\left(\frac{t}{2} \theta^{p}\left(\varepsilon_{1}\right)+\left(\frac{2-t}{2}\right) \theta^{p}\left(\varepsilon_{2}\right)\right)^{1 / p}\right) \mathrm{d} t \\
& \quad+\int_{0}^{1} t^{\tau-1} E_{\sigma, \tau, \delta}^{\rho, r, k, c}\left(\omega t^{\sigma} ; \bar{p}\right) \varphi\left(\left(\frac{t}{2} \theta^{p}\left(\varepsilon_{2}\right)+\left(\frac{2-t}{2}\right) \theta^{p}\left(\varepsilon_{1}\right)\right)^{1 / p}\right) h\left(\left(\frac{t}{2} \theta^{p}\left(\varepsilon_{1}\right)+\left(\frac{2-t}{2}\right) \theta^{p}\left(\varepsilon_{2}\right)\right)^{1 / p}\right) \mathrm{d} t .
\end{aligned}
$$

By choosing $\theta(x)=(t / 2) \theta^{p}\left(\varepsilon_{1}\right)+((2-t) / 2) \theta^{p}\left(\varepsilon_{2}\right)$, that is, $\theta^{P}\left(\varepsilon_{1}\right)+\theta^{P}\left(\varepsilon_{2}\right)-\theta(x)=(t / 2) \theta^{p}\left(\varepsilon_{2}\right)+((2-t) / 2) \theta^{p}\left(\varepsilon_{1}\right)$, in (49) and using the condition $\varphi\left(\theta^{1 / p}(x)\right)=\varphi \quad\left(\left(\theta^{p}\right.\right.$ $\left.\left.\left(\varepsilon_{1}\right)+\theta^{p}\left(\varepsilon_{2}\right)-\theta(x)\right)^{1 / p}\right)$, one can get first inequality of $(47)$.
To prove the second inequality of (47), multiplying both sides of (45) by $t^{\tau-1} E_{\sigma, \tau, \delta}^{\rho, r, k, c}\left(\omega t^{\sigma} ; \bar{p}\right) h\left(\left((t / 2) \quad \theta^{P}\left(\varepsilon_{1}\right)+\right.\right.$ $\left.\left.((2-t) / 2) \theta^{p}\left(\varepsilon_{2}\right)\right)^{1 / p}\right)$ and then integrating over $[0,1]$, we have 


$$
\begin{aligned}
& \int_{0}^{1} t^{\tau-1} E_{\sigma, \tau, \delta}^{\rho, r, k, c}\left(\omega t^{\sigma} ; \bar{p}\right) \varphi\left(\left(\frac{t}{2} \theta^{p}\left(\varepsilon_{1}\right)+\left(\frac{2-t}{2}\right) \theta^{p}\left(\varepsilon_{2}\right)\right)^{1 / p}\right) h\left(\left(\frac{t}{2} \theta^{p}\left(\varepsilon_{1}\right)+\left(\frac{2-t}{2}\right) \theta^{p}\left(\varepsilon_{2}\right)\right)^{1 / p}\right) \mathrm{d} t \\
& \quad+\int_{0}^{1} t^{\tau-1} E_{\sigma, \tau, \delta}^{\rho, r, k, c}\left(\omega t^{\sigma} ; \bar{p}\right) \varphi\left(\left(\frac{t}{2} \theta^{p}\left(\varepsilon_{2}\right)+\left(\frac{2-t}{2}\right) \theta^{p}\left(\varepsilon_{1}\right)\right)^{1 / p}\right) h\left(\left(\frac{t}{2} \theta^{p}\left(\varepsilon_{1}\right)+\left(\frac{2-t}{2}\right) \theta^{p}\left(\varepsilon_{2}\right)\right)^{1 / p}\right) \mathrm{d} t \\
& \leq\left(\varphi\left(\theta\left(\varepsilon_{1}\right)\right)+\varphi\left(\theta\left(\varepsilon_{2}\right)\right)\right) \\
& \quad \cdot \int_{0}^{1} t^{\tau-1} E_{\sigma, \tau, \delta}^{\rho, r, k, c}\left(\omega t^{\sigma} ; \bar{p}\right) h\left(\left(\frac{t}{2} \theta^{p}\left(\varepsilon_{1}\right)+\left(\frac{2-t}{2}\right) \theta^{p}\left(\varepsilon_{2}\right)\right)^{1 / p}\right) \mathrm{d} t .
\end{aligned}
$$

Setting $\theta(x)=(t / 2) \theta^{p}\left(\varepsilon_{1}\right)+((2-t) / 2) \theta^{p}\left(\varepsilon_{2}\right)$ in (38) and using the condition $\varphi\left(\theta^{1 / p}(x)\right)=\varphi\left(\left(\theta^{p}\left(\varepsilon_{1}\right)+\theta^{p}\left(\varepsilon_{2}\right)-\theta(x)\right)^{1 / p}\right)$, one can get second inequality of (47).

(ii) Proof is similar to the proof of (i).

\section{Remark 5}

(i) By setting $p=-1, \bar{p}=0$, and $\theta=I$, Theorem 2.6 of [22] is obtained. (ii) By setting $\theta=I$ and $p=-1$, Theorem 2.6 of [23] is obtained.

(iii) By setting $p=-1$, Theorem 2.10 of [24] is obtained.

Corollary 3. When we set $p=-1, \omega=\bar{p}=0$, and $\theta=I$ in Theorem 6, then we get the following inequalities via $R L$ fractional integrals.

$$
\begin{aligned}
& \varphi\left(\frac{2 \varepsilon_{1} \varepsilon_{2}}{\varepsilon_{1}+\varepsilon_{2}}\right)\left(\left(I_{\left(\left(\varepsilon_{1}+\varepsilon_{2}\right) / 2 \varepsilon_{1} \varepsilon_{2}\right)^{+}}^{\tau} \circ \psi\right)\left(\frac{1}{\varepsilon_{1}}\right)+\left(I_{\left(\left(\varepsilon_{1}+\varepsilon_{2}\right) / 2 \varepsilon_{1} \varepsilon_{2}\right)^{-}}^{\tau} \circ \psi\right)\left(\frac{1}{\varepsilon_{2}}\right)\right) \\
& \leq\left(I^{\tau}\left(\left(\varepsilon_{1}+\varepsilon_{2}\right) / 2 \varepsilon_{1} \varepsilon_{2}\right)^{+} \varphi h \circ \psi\right)\left(\frac{1}{\varepsilon_{1}}\right)+\left(I_{\left(\left(\varepsilon_{1}+\varepsilon_{2}\right) / 2 \varepsilon_{1} \varepsilon_{2}\right)}^{\tau} \varphi h \circ \psi\right)\left(\frac{1}{\varepsilon_{2}}\right) \\
& \leq \frac{\varphi\left(\varepsilon_{1}\right)+\varphi\left(\varepsilon_{2}\right)}{2}\left(\left(I_{\left(\left(\varepsilon_{1}+\varepsilon_{2}\right) / 2 \varepsilon_{1} \varepsilon_{2}\right)^{+}}^{\tau} \circ \circ \psi\right)\left(\frac{1}{\varepsilon_{1}}\right)+\left(I_{\left(\left(\varepsilon_{1}+\varepsilon_{2}\right) / 2 \varepsilon_{1} \varepsilon_{2}\right)^{-}}^{\tau} \circ \psi\right)\left(\frac{1}{\varepsilon_{2}}\right)\right) \text {. }
\end{aligned}
$$

\section{Conclusion}

We have established Hadamard and Fejér-Hadamard fractional integral inequalities for generalized fractional integrals of $p$-convex functions. The results of this paper hold simultaneously for convex and harmonically convex functions for different fractional integral operators containing Mittag-Leffler functions.

\section{Data Availability}

No data were used to support this study.

\section{Conflicts of Interest}

The authors declare that they have no conflicts of interest.

\section{References}

[1] M. Andrić, G. Farid, and J. Pečarić, "A further extension of Mittag-Leffler function," Fractional Calculus and Applied Analysis, vol. 21, no. 5, pp. 1377-1395, 2018.
[2] G. Farid, "A unified integral operator and further its consequences," Open Journal of Mathematical Analysis, vol. 4, no. 1, pp. $1-7,2020$.

[3] Y. C. Kwun, G. Farid, S. Ullah, W. Nazeer, K. Mahreen, and S. M. Kang, "Inequalities for a unified integral operator and associated results in fractional calculus," IEEE Access, vol. 7, pp. 126283-126292, 2019.

[4] T. O. Salim and A. W. Faraj, "A Generalization of MittagLeffler function and integral operator associated with integral calculus," J. Frac. Calc. Appl, vol. 3, no. 5, pp. 1-13, 2012.

[5] G. Rahman, D. Baleanu, M. A. Qurashi, S. D. Purohit, S. Mubeen, and M. Arshad, "The extended generalized MittagLeffler function via fractional calculus," Journal of Nonlinear Sciences and Applications, vol. 10, no. 1, pp. 4244-4253, 2017.

[6] H. M. Srivastava and Ž. Tomovski, "Fractional calculus with an integral operator containing a generalized Mittag-Leffler function in the kernel," Applied Mathematics and Computation, vol. 211, no. 1, pp. 198-210, 2009.

[7] T. R. Prabhakar, "A singular integral equation with a generalized Mittag-Leffler function in the kernel," Yokohama Mathematical Journal, vol. 19, pp. 7-15, 1971.

[8] Z. Tomovski, R. Hiller, and H. M. Srivastava, "Fractional and operational calculus with generalized fractional derivative operators and Mittag-Leffler function," Integral Transforms and Special Functions, vol. 21, no. 11, pp. 797-814, 2011. 
[9] G. H. Toader, "Some generalization of convexity," Proceedings of the Colloquium on Approximation and Optimization. ClujNapoca (Romania), University of Cluj-Napoca, Cluj-Napoca, pp. 329-338, 1984.

[10] I. Iscan, "Hermite-Hadamard type inequalities for harmonically convex functions," Hacettepe Journal of Mathematics and Statistics, vol. 43, no. 6, pp. 935-942, 2014.

[11] M. Kunt and İ. İşcan, "Hermite-Hadamard-Fejér type inequalities for $p$-convex functions via fractional integrals, Iran," Iranian Journal of Science and Technology, Transactions A: Science, vol. 42, no. 4, pp. 2079-2089, 2018.

[12] L. Fejér, "Überdie Fourierreihen II," Math Naturwiss Anz Ungar Akad Wiss, vol. 24, pp. 369-390, 1906.

[13] B. Ahmad, A. Alsaedi, M. Kirane, and B. T. Torebek, "Hermite-Hadamard, Hermite-Hadamard-Fejér, DragomirAgarwal and Pachpatte type inequalities for convex functions via new fractional integrals," Journal of Computational and Applied Mathematics, vol. 353, pp. 120-129, 2019.

[14] M. K. Bakula and J. Pečarić, "Note on some Hadamard-type inequalties," Journal of Inequalities in Pure and Applied Mathematics, vol. 5, no. 3, p. 74, 2004.

[15] H. Chen and U. N. Katugampola, "Hermite-Hadamard and Hermite-Hadamard-Fejér type inequalities for generalized fractional integrals," Journal of Mathematical Analysis and Applications, vol. 446, no. 2, pp. 1274-1291, 2017.

[16] F. Chen and S. Wu, "Fejér and Hermite-Hadamard type Inequalities for harmonically convex functions," Journal of Applied Mathematics, vol. 2014, Article ID 386806, 2014.

[17] I. Iscan, "Ostrowski type inequalities for $p$-convex functions," New Trends in Mathematical Science, vol. 4, no. 3, p. 140, 2016.

[18] I. Iscan and $\mathrm{S}$. Wu, "Hemite-Hadamard type inequalities for harmonically convex functions via fractional integrals," Applied Mathematics and Computation, vol. 238, pp. 237-244, 2014.

[19] S. Kang, G. Abbas, G. Farid, and W. Nazeer, "A generalized Fejér-Hadamard inequality for harmonically convex functions via generalized fractional integral operator and related results," Mathematics, vol. 6, no. 7, p. 122, 2018.

[20] M. Kunt, I. Iscan, N. Yazi, and U. Gozutok, "On new inequalities of Hermite-Hadamard-Fejér type inequalities for harmonically convex functions via fractional integrals," Springer Plus, vol. 5, no. 1, pp. 1-19, 2016.

[21] Y. Rao, M. Yussouf, G. Farid, J. Pečarić, and I. Tlili, "Further generalizations of Hadamard and Fejér-Hadamard inequalities and error estimations," Advances in Difference Equations, vol. 2020, p. 421, 2020.

[22] G. Abbas and G. Farid, "Hadamard and Fejér-Hadamard type inequalities for harmonically convex functions via generalized fractional integrals," The Journal of Analysis, vol. 25, no. 1, pp. 107-119, 2017.

[23] G. Farid, A. U. Rehman, and S. Mehmood, "Hadamard and Fejér-Hadamard type integral inequalities for harmonically convex functions via an extended generalized Mittag-Leffler function," Journal of Mathematics and Computer Science, vol. 8, no. 5, pp. 630-643, 2018.

[24] X. Qiang, G. Farid, M. Yussouf, K. A. Khan, and A. U. Rehman, "New generalized fractional versions of Hadamard and Fejér inequalities for harmonically convex functions," Journal of Inequalities and Applications, vol. 2020, p. 191, 2020.

[25] G. Farid and G. Abbas, "Generalizaions of some HermiteHadamard-Fejér type inequalities for $p$-convex functions via generalized fractional integrals," Journal of Fractional Calculus and Applications, vol. 9, no. 2, pp. 56-76, 2018. 